MATHEMATICS OF COMPUTATION

Volume 70, Number 234, Pages 607-636

S 0025-5718(00)01209-6

Article electronically published on March 2, 2000

\title{
OPTIMAL ORDER COLLOCATION FOR THE MIXED BOUNDARY VALUE PROBLEM ON POLYGONS
}

\author{
PASCAL LAUBIN
}

\begin{abstract}
In usual boundary elements methods, the mixed Dirichlet-Neumann problem in a plane polygonal domain leads to difficulties because of the transition of spaces in which the problem is well posed. We build collocation methods based on a mixed single and double layer potential. This indirect method is constructed in such a way that strong ellipticity is obtained in high order spaces of Sobolev type. The boundary values of this potential define a bijective boundary operator if a modified capacity adapted to the problem is not 1 . This condition is analogous to the one met in the use of the single layer potential, and is not a problem in practical computations. The collocation methods use smoothest splines and known singular functions generated by the corners. If splines of order $2 m-1$ are used, we get quasi-optimal estimates in $H^{m}$-norm. The order of convergence is optimal in the sense that it is fixed by the approximation properties of the first missed singular function.
\end{abstract}

\section{INTRODUCTION}

The resolution of elliptic problems by boundary elements techniques already has a long history, see for example [20. If the boundary is smooth and there is only one boundary condition, stability and convergence have been proved in rather general settings for Galerkin and collocation methods. Collocation methods are the most widely spread in practical computations because of their simplicity and their efficiency. However, stability and convergence are not automatic, and are more difficult to prove than for Galerkin methods. Recent works are concerned with the collocation methods for nonsmooth boundaries and use different strategies to deal with the singularities of the solution generated by the irregularities of the boundary. In [7] and [14], optimal rates of convergence have been obtained for the Dirichlet problem in a plane polygonal domain.

In this paper, we build collocation methods for the mixed Dirichlet-Neumann problem, which is a widespread model in several domains of mathematical physics. A classical reference for numerical methods in this problem is [3]. In that paper, Costabel and Stephan use the direct Fix method to build Galerkin procedures. The boundary unknowns are the parts of the Dirichlet and Neumann data which are not given. The main point is to prove the strong ellipticity of the numerical schemes. The difficulty comes essentially from the transition of the spaces where

Received by the editor May 22, 1998 and, in revised form, November 17, 1998 and March 16, 1999.

2000 Mathematics Subject Classification. Primary 65N35, 65R20; Secondary 45B05.

Key words and phrases. Collocation method, mixed problem, optimal order.

(C)2000 American Mathematical Society 
the problem is well-posed, $H^{1 / 2}$ on the Dirichlet part of the boundary and $H^{-1 / 2}$ on the Neumann part.

The pseudodifferential calculus which reduces this condition to the positivity of a symbol cannot be used, since the boundary is not smooth and since there are changes in the boundary conditions. The Mellin transform leads to a complex symbol without a good positivity property. The coerciveness of the bilinear form defining the energy norm of the variational formulation cannot be used, since it is not defined on the spaces used by the Galerkin method. To avoid this problem, the authors perform a Gaussian type elimination procedure near each mixed corner on the matrix of boundary operators they use. This problem leads to a modified method involving compositions of boundary operators. Coerciveness is guaranteed in $L^{2}$ but not for stronger norms. This limits the orders of convergence independently of the choice of the test functions and of the number of singular functions used at the corners. Some higher orders of convergence can be obtained in Sobolev spaces with negative indices, see [5].

Another approach is presented in 8. The authors use the single layer potential and cosine approximation spaces, and build collocation methods based on a mesh grading transformation procedure. Using an intricate Mellin analysis, the authors prove convergence with a rate as high as allowed by the mesh grading. However, some invertibility in $L^{p}$-spaces for any $p>1$ is assumed, and stability is proved only if the possibility is allowed of cutting off by zero the approximate solution in some intervals near each corner.

Here, we construct an optimal order Fix collocation method without mesh grading and with full numerical stability. It is based on smoothest splines. One new tool is an indirect method based on a modified ansatz (3) for the boundary potential. The main results of [2] show that it defines an $H^{1}$ function if the data are in suitable Sobolev spaces and the data on the Dirichlet part of the boundary vanishes at the mixed corners. It is chosen in such a way that coerciveness holds in spaces with high regularity. It consists of a single layer potential on the Neumann part of the boundary and of a double layer potential on the Dirichlet part. Moreover, in the collocation equations, we use the tangential derivative of the unknown on the Dirichlet part. This method can be applied to the interior problem and to the exterior one.

Since our method is not the direct one nor a classical one, we have to prove the bijectivity of the boundary operator that we obtain. It turns out that the vanishing condition required on the Dirichlet part in the ansatz is balanced by an additional singular function appearing at each mixed corner. Contrary to the case of the pure Dirichlet or Neumann problem, we get boundary unknowns which are not $L^{2}$. However, they have the $H^{-1 / 2}$ regularity which is the limit allowed by the variational setting. The study of the invertibility leads to a condition involving a modified capacity associated to the open set and the decomposition of the boundary. This condition is similar to the one met in the use of the single layer potential for solving the Dirichlet problem.

The next section contains the description of the method and the main results. Section 3 describe the variational setting of the problem and some properties of the $H^{1 / 2}$ spaces. In Section 4 we adapt the notion of capacity to the mixed problem The local Mellin analysis near the corners is performed in Section [5 In Section 6] we prove that the boundary operator is bijective when the mixed capacity is not 1 . 
Section $[7$ is devoted to the proofs of the main theorems. The last section presents numerical tests of the method.

\section{Main RESUlts AND NUmerical Methods}

Let us consider a bounded polygonal open subset $\Omega$ of $\mathbb{R}^{2}$ with a connected boundary

$$
\Gamma=\partial \Omega=\bigcup_{j=0}^{M-1} \Gamma_{j} .
$$

Here $\Gamma_{j}, 0 \leq j<M$, is a closed straight line segment. For convenience, we define $\Gamma_{j+M}=\Gamma_{j}$ for any $j$. We denote by $P_{j}$ the corner point where $\Gamma_{j-1}$ and $\Gamma_{j}$ meet. The interior angle at $P_{j}$ is denoted by $\omega_{j}$. It is assumed that this angle belongs to $] 0,2 \pi[\backslash\{\pi\}$. Denote by $\nu$ the unit inward normal vector and by $t$ the unit tangent vector on the boundary. They exist outside the corners. We fix the orientation in such a way that $(t, \nu)$ is a positive basis. In the definitions of the singular exponents and of the associated spaces and operators, we use the index + for the interior domain and - for the exterior one.

Assume that we have a decomposition $\Gamma=\Gamma_{D} \cup \Gamma_{N}$, where $\Gamma_{D}=\bigcup_{j \in e_{D}} \Gamma_{j}$, $\Gamma_{N}=\bigcup_{j \in e_{N}} \Gamma_{j}, e_{D} \cap e_{N}=\emptyset$ and $e_{D} \cup e_{N}=\{0,1, \ldots, M-1\}$. We assume that $e_{N} \neq \emptyset$ since this simplifies the exposition. The pure Dirichlet problem has already been considered by a similar method in 14 .

We denote by $e_{N D}$ the set of indices $j \in e_{D}$ such that $j-1(M-1$ if $j=0)$ belongs to $e_{N}$. Let $p$ be the number of elements of $e_{N D}$. It follows that $\Gamma_{D}$ has $p$ connected components $\Gamma_{D, 1}, \ldots, \Gamma_{D, p}$.

We consider the interior mixed Dirichlet-Neumann problem

$$
\begin{cases}-\Delta u=0 & \text { in } \quad \Omega, \\ u_{\mid \Gamma_{D}}=u_{0}, & \partial_{\nu} u_{\mid \Gamma_{N}}=u_{1},\end{cases}
$$

and also the exterior problem

$$
\left\{\begin{array}{l}
-\Delta u=0 \quad \text { in } \quad \mathbb{R}^{2} \backslash \bar{\Omega}, \\
u_{\mid \Gamma_{D}}=u_{0}, \quad \partial_{\nu} u_{\mid \Gamma_{N}}=u_{1}, \\
u(x)=a \log |x|+O(1), \quad x \rightarrow \infty,
\end{array}\right.
$$

with a singularity at infinity. Here $u_{0}, u_{1}$ and $a$ are given. This formulation of the exterior problem with $a \neq 0$ contains the Green's function with pole at infinity. It is useful for the presentation of our results below.

If $\Gamma_{D}$ is not empty, these problems have a unique variational solution $u$ for any data $u_{0} \in H^{1 / 2}\left(\Gamma_{D}\right), u_{1} \in H^{-1 / 2}\left(\Gamma_{N}\right)$ and $a \in \mathbb{C}$. If $\Gamma_{D}=\emptyset$, then the solution exists and is unique modulo a constant if $u_{1} \in H^{-1 / 2}\left(\Gamma_{N}\right)$ has mean value 0 in the interior case and satisfies

$$
a+\frac{1}{2 \pi} \int_{\Gamma} u_{1} d \sigma=0
$$

in the exterior case. 
2.1. The spaces. Let $s \geq 0$. With $C=\Gamma, \Gamma_{D}$ or $\Gamma_{N}$, we consider the usual Sobolev spaces

$$
H^{s}(C)=\left\{u_{\mid C}: u \in H^{s+1 / 2}\left(\mathbb{R}^{2}\right)\right\}
$$

and the spaces of functions that have the $H^{s}$-regularity on each side without continuity at the corners

$$
H_{c}^{s}(C)=\left\{f \in L^{2}(C): f \circ \gamma_{j} \in H^{s}(] 0, t_{j}[), j \in e_{C}\right\}
$$

with $e_{C}=\{0,1, \ldots, M-1\}, e_{D}$ or $e_{N}$, respectively. Here $\gamma_{j}:\left[0, t_{j}\right] \rightarrow \Gamma_{j}$ is the parameterization by arc length. We use the norm

$$
\|f\|_{H_{c}^{s}(C)}^{2}=\sum_{j \in e_{C}}\left\|f_{\mid \Gamma_{j}} \circ \gamma_{j}\right\|_{H^{s}(] 0, t_{j}[)}^{2} .
$$

We also consider

$$
H_{0}^{s}(C)=\left\{f \in H^{s}(C): f_{\mid \Gamma_{j}} \in H_{0}^{s}\left(\Gamma_{j}\right), j \in e_{C}\right\}
$$

with the induced norms, where

$$
H_{0}^{s}\left(\Gamma_{j}\right)=\left\{f \in L^{2}\left(\Gamma_{j}\right): f \circ \gamma_{j} \in H_{0}^{s}(] 0, t_{j}[)\right\}
$$

and $H_{0}^{s}(] 0, t_{j}[)$ is the closure of $C_{0}^{\infty}(] 0, t_{j}[)$ in $H^{s}(] 0, t_{j}[)$.

The case $s=\frac{1}{2}$ is of particular use in our constructions, and its special nature requires some care. See for example [10] for basic properties and some characterizations of $H^{1 / 2}(\mathbb{R})$.

Following [3], we define $\widetilde{H}^{1 / 2}(C)$ as the set of elements of $L^{2}(C)$ whose extensions by 0 outside $C$ belong to $H^{1 / 2}(\Gamma)$. Of course $\widetilde{H}^{1 / 2}(\Gamma)=H^{1 / 2}(\Gamma)$. We also need the dual spaces

$$
\widetilde{H}^{-1 / 2}(C)=H^{1 / 2}(C)^{\prime}
$$

and

$$
H^{-1 / 2}(C)=\widetilde{H}^{1 / 2}(C)^{\prime} .
$$

We define $\widetilde{H}_{v}^{-1 / 2}(C)$ as the subspace of $\widetilde{H}^{-1 / 2}(C)$ formed by the elements whose integrals on each connected component of $C$ vanish.

The previous spaces have to be refined to take into account the singularities generated by the corners. The singular exponents can be defined in the following way. They are close to the ones described in [10, but some modifications occur since we are considering singularities of boundary data and not of the solution itself.

For a mixed corner $P$ with interior angle $\omega$, let

$$
\begin{gathered}
e_{\omega, e}^{(m)}=\left\{\left(2 k-\frac{1}{2}\right) \frac{\pi}{\omega}-1: k \in \mathbb{N} \backslash\{0\}\right\} \cup\left\{\left(2 k-\frac{1}{2}\right) \frac{\pi}{2 \pi-\omega}-1: k \in \mathbb{N} \backslash\{0\}\right\}, \\
e_{\omega, o}^{(m)}=\left\{\left(2 k+\frac{1}{2}\right) \frac{\pi}{\omega}-1: k \in \mathbb{N}\right\} \cup\left\{\left(2 k+\frac{1}{2}\right) \frac{\pi}{2 \pi-\omega}-1: k \in \mathbb{N}\right\}
\end{gathered}
$$

and

$$
e_{\omega}^{(m)}=e_{\omega, e}^{(m)} \cup e_{\omega, o}^{(m)}
$$

If $s-\frac{1}{2} \notin e_{\omega}^{(m)}$, denote by $\mathcal{L}_{\omega, s, \pm}^{(m)}$ the linear hull in $L^{2}(\Gamma)$ of the functions

- $\left(\begin{array}{c}x^{\alpha} \\ \pm x^{\alpha}\end{array}\right)$ if $\alpha \in e_{\omega, e}^{(m)},-1<\alpha<s-\frac{1}{2}$ 
- $\left(\begin{array}{c}x^{\alpha} \\ \pm x^{\alpha}\end{array}\right) \ln (x)$ if $\alpha \in e_{\omega, e}^{(m)},-1<\alpha<s-\frac{1}{2}$ and $\alpha \in \frac{1}{2}+\mathbb{N}$,

- $\left(\begin{array}{c}x^{\alpha} \\ \mp x^{\alpha}\end{array}\right)$ if $\alpha \in e_{\omega, o}^{(m)},-1<\alpha<s-\frac{1}{2}$,

- $\left(\begin{array}{c}x^{\alpha} \\ \mp x^{\alpha}\end{array}\right) \ln (x)$ if $\alpha \in e_{\omega, o}^{(m)},-1<\alpha<s-\frac{1}{2}$ and $\alpha \in \frac{1}{2}+\mathbb{N}$,

where $x$ is the distance to $P$. Here, the vector notation means that the first component is the value of the function on the segment preceding $P$ in the direction of the tangent vector $t$ and the second one is the value on the segment following $P$. The function is equal to zero on the other sides.

Note also that if $n$ is an integer and $n-1 / 2 \notin e_{\omega}^{(m)}$, then $\operatorname{dim} \mathcal{L}_{\omega, n, \pm}^{(m)}=2 n+1$.

If $P_{j}$ is a mixed corner, the set of singular exponents is $e_{\omega_{j}}=e_{\omega_{j}}^{(m)}$. The associated singular functions space is $\mathcal{L}_{j, s}^{ \pm}=\mathcal{L}_{\omega_{j}, s, \pm}^{(m)}$ if $j-1 \in e_{N}$ and $j \in e_{D}$. In the other case, $j-1 \in e_{D}$ and $j \in e_{N}$, we have to use $\mathcal{L}_{j, s}^{ \pm}=\mathcal{L}_{\omega_{j}, s, \mp}^{(m)}$. We remind the reader that the + (resp. - ) corresponds to the interior (resp. exterior) problem.

We proceed in the same way for the pure Dirichlet and Neumann corners. Let

$$
\begin{gathered}
e_{\omega, e}^{(p)}=\left\{\frac{2 k \pi}{\omega}-1: k \in \mathbb{N}\right\} \cup\left\{\frac{(2 k+1) \pi}{2 \pi-\omega}-1: k \in \mathbb{N}\right\}, \\
e_{\omega, o}^{(p)}=\left\{\frac{(2 k+1) \pi}{\omega}-1: k \in \mathbb{N}\right\} \cup\left\{\frac{2 k \pi}{2 \pi-\omega}-1: k \in \mathbb{N}\right\}
\end{gathered}
$$

and $e_{\omega}^{(p)}=e_{\omega, e}^{(p)} \cup e_{\omega, o}^{(p)}$. If $s-\frac{1}{2} \notin e_{\omega}^{(p)}$, denote by $\mathcal{L}_{\omega, s, \pm}^{(p)}$ the linear hull in $L^{2}(\Gamma)$ of the functions

- $\left(\begin{array}{c}x^{\alpha} \\ \pm x^{\alpha}\end{array}\right)$ if $\alpha \in e_{\omega, e}^{(p)},-\frac{1}{2}<\alpha<s-\frac{1}{2}$

- $\left(\begin{array}{c}x^{\alpha} \\ \pm x^{\alpha}\end{array}\right) \ln (x)$ if $\alpha \in e_{\omega, e}^{(p)}, 0<\alpha<s-\frac{1}{2}$ and $\alpha \in \frac{1}{2}+\mathbb{N}$

- $\left(\begin{array}{c}x^{\alpha} \\ \mp x^{\alpha}\end{array}\right)$ if $\alpha \in e_{\omega, o}^{(p)},-\frac{1}{2}<\alpha<s-\frac{1}{2}$,

- $\left(\begin{array}{c}x^{\alpha} \\ \mp x^{\alpha}\end{array}\right) \ln (x)$ if $\alpha \in e_{\omega, o}^{(p)}, 0<\alpha<s-\frac{1}{2}$ and $\alpha \in \frac{1}{2}+\mathbb{N}$

with the same notations as above.

Clearly $-1 / 2 \notin e_{\omega}^{(m)}$ if $\left.\omega \in\right] 0,2 \pi\left[\backslash\{\pi\}\right.$. Hence $\operatorname{dim} \mathcal{L}_{\omega, 0, \pm}^{(m)}=1$. This one dimensional space is not included in $L^{2}(\Gamma)$. However it is included in $H^{-1 / 2}(\Gamma)$, since $x^{\alpha} \in H^{-1 / 2}\left(\mathbb{R}_{+}\right)$if $-1<\alpha<-1 / 2$.

Note that if $n$ is an integer and $n-\frac{1}{2} \notin e_{\omega}^{(p)}$, then $\operatorname{dim} \mathcal{L}_{\omega, n, \pm}^{(p)}=2 n$. Hence there is a shift in the dimension of the spaces of singular functions between the two types of corners.

If $P_{j}$ is a pure Dirichlet or Neumann corner, then the set of singular exponents is $e_{\omega_{j}}=e_{\omega_{j}}^{(p)}$ and the associated singular functions space is $\mathcal{L}_{j, s}^{ \pm}=\mathcal{L}_{\omega_{j}, s, \pm}^{(p)}$.

For each $j$, choose a function $\chi_{j}$ on $\Gamma$ which is the restriction of an element of $C_{0}^{\infty}\left(\mathbb{R}^{2}\right)$ and equal to $\delta_{j k}$ near $P_{k}$ for every $k$. Denote by $\mathcal{H}_{ \pm}^{s}\left(\Gamma_{D}, \Gamma_{N}\right)$ the subspace of

$$
H_{0}^{s}\left(\Gamma_{D}\right) \times H_{0}^{s}\left(\Gamma_{N}\right)+\sum_{j=0}^{M-1} \chi_{j} \mathcal{L}_{j, s}^{ \pm}
$$


formed by the elements $(f, g)$ such that the integral of $f$ on each connected component of $\Gamma_{D}$ is 0 . This space does not depend on the choice of the functions $\chi_{j}$.

Note that $\mathcal{H}_{ \pm}^{s}\left(\Gamma_{D}, \Gamma_{N}\right)$ is not a subspace $L^{2}\left(\Gamma_{D}\right) \times L^{2}\left(\Gamma_{N}\right)$ if there is at least one mixed corner $P_{j}$. Indeed, $-1 / 2 \notin e_{\omega}^{(m)}$ if $\left.\omega \in\right] 0,2 \pi\left[\backslash\{\pi\}\right.$. Hence $\operatorname{dim} \mathcal{L}_{j, 0}^{ \pm}=1$. The one dimensional space $\chi_{j} \mathcal{L}_{j, 0}^{ \pm}=1$ is not included in $L^{2}(\Gamma)$. However it is included in $H^{-1 / 2}(\Gamma)$, since $x^{\alpha}$ belongs to $H^{-1 / 2}\left(\mathbb{R}_{+}\right)$if $-1<\alpha<-1 / 2$. This additional degree of freedom in the unknown function at the mixed corners is balanced by the requirement that the integral of $f$ vanishes on each connected component of $\Gamma_{D}$.

We can of course see the elements of $\mathcal{H}_{ \pm}^{s}\left(\Gamma_{D}, \Gamma_{N}\right)$ as a pair of functions, one on $\Gamma_{D}$ and one on $\Gamma_{N}$, or as a single function on $\Gamma$. For every $j$, let $u_{j k}, 0 \leq k<K_{j}$, be a basis of $\mathcal{L}_{j, s}^{ \pm}$. If

$$
u=v+\sum_{j=0}^{M-1} \chi_{j} \sum_{k=0}^{K_{j}-1} c_{j k} u_{j k}
$$

with $v \in H_{0}^{s}\left(\Gamma_{D}\right) \times H_{0}^{s}\left(\Gamma_{N}\right)$, then $\mathcal{H}_{ \pm}^{s}\left(\Gamma_{D}, \Gamma_{N}\right)$ is a Hilbert space for the norm

$$
\|u\|_{\mathcal{H}_{ \pm}^{s}\left(\Gamma_{D}, \Gamma_{N}\right)}^{2}=\sum_{j=0}^{M-1} \sum_{k=0}^{K_{j}-1}\left|c_{j k}\right|^{2}+\|v\|_{H_{0}^{s}\left(\Gamma_{D}\right) \times H_{0}^{s}\left(\Gamma_{N}\right)}^{2} .
$$

The choice of the basis of singular functions does not matter for the asymptotic estimates, since the space is finite dimensional. However, a well designed basis close to orthogonality is important for the condition number in practical computations.

2.2. The boundary operators. To solve (1), we use the following ansatz:

$$
K(g, h)(x)=\frac{1}{2 \pi} \int_{\Gamma_{N}} g(y) \log |x-y| d \sigma(y)+\frac{1}{2 \pi} \int_{\Gamma_{D}} \frac{(x-y) \cdot \nu_{y}}{|x-y|^{2}} h(y) d \sigma(y)
$$

for $x \in \mathbb{R}^{2} \backslash \Gamma$. It is quite different from the one used in the direct method. In this case the two previous integrals contain the known boundary data $u_{1}$ and $u_{0}$, and the boundary unknowns appear in the single layer potential on $\Gamma_{D}$ and the double layer potential on $\Gamma_{N}$.

To solve (2), we use the similar ansatz

$$
K(g, h, c)(x)=c+\frac{1}{2 \pi} \int_{\Gamma_{N}} g(y) \log |x-y| d \sigma(y)+\frac{1}{2 \pi} \int_{\Gamma_{D}} \frac{(x-y) \cdot \nu_{y}}{|x-y|^{2}} h(y) d \sigma(y)
$$

for $x \in \mathbb{R}^{2} \backslash \Gamma$. The constant $c$ is required to get surjectivity of the boundary operators defined below.

Let $(f, g) \in \widetilde{H}_{v}^{-1 / 2}\left(\Gamma_{D}\right) \times \widetilde{H}^{-1 / 2}\left(\Gamma_{N}\right)$. By Corollary 5 , there is a unique $h \in$ $\widetilde{H}^{1 / 2}\left(\Gamma_{D}\right)$ such that $\partial_{t} h=f$. By Theorem 1 of [2], the function (3) defined by $g$ and $h$ belongs to $H^{1}(\Omega)$. In the same way, the function (4) is $H^{1}$ in any bounded open subset of $\mathbb{R}^{2} \backslash \bar{\Omega}$ and has the asymptotic behavior required in (2).

We consider the operators

$$
\begin{aligned}
T_{ \pm}: \widetilde{H}_{v}^{-1 / 2}\left(\Gamma_{D}\right) \times \widetilde{H}^{-1 / 2}\left(\Gamma_{N}\right) & \rightarrow H^{-1 / 2}\left(\Gamma_{D}\right) \times H^{-1 / 2}\left(\Gamma_{N}\right), \\
(f, g) & \rightarrow\left(\partial_{t} u_{\mid \Gamma_{D}}, \partial_{\nu} u_{\mid \Gamma_{N}}\right),
\end{aligned}
$$


where $u$ is defined by (31) for the interior problem, by (4) for the exterior one, and $h \in \widetilde{H}^{1 / 2}\left(\Gamma_{D}\right)$ satisfies $\partial_{t} h=f$. The subscript $+($ resp. -$)$ means that we consider the interior (resp. exterior) problem and that the boundary values are taken from inside (resp. outside).

The existence of the boundary values and of the normal derivative in the space $H^{1 / 2}\left(\Gamma_{D}\right) \times H^{-1 / 2}\left(\Gamma_{N}\right)$ is a consequence of Lemmas 3.2 and 3.6 of [2], since $u$ is in the maximal domain of $\Delta$. This shows that the operators $T_{ \pm}$make sense, since the tangential derivative maps $H^{1 / 2}\left(\Gamma_{D}\right)$ into $H^{-1 / 2}\left(\Gamma_{D}\right)$ (see for example [10]).

If $(f, g) \in L^{2}\left(\Gamma_{D}\right) \times L^{2}\left(\Gamma_{N}\right)$, it follows from the results of [12] and [13] that the function $u$ defined by (3) belongs to $H^{3 / 2}(\Omega)$ and that the one defined by (4) is $H^{3 / 2}$ in any bounded open subset of $\mathbb{R}^{2} \backslash \bar{\Omega}$. Hence the traces are continuous on the closure of each side $\Gamma_{j}$. This remains true if $(f, g) \in \mathcal{H}_{ \pm}^{0}\left(\Gamma_{D}, \Gamma_{N}\right)$, but then it is a consequence of these results and of Proposition 7 .

For each $j=1, \ldots, p$, we fix a point $Q_{j}$ on $\Gamma_{D, j}$. We can for example take the points $P_{j}$ with $j \in e_{N D}$. We use the finite rank operators

$$
\begin{gathered}
S_{+}: \mathcal{H}_{+}^{0}\left(\Gamma_{D}, \Gamma_{N}\right) \rightarrow \mathbb{C}^{p}:(f, g) \mapsto\left(u\left(Q_{1}\right), \ldots, u\left(Q_{p}\right)\right), \\
S_{-}: \mathcal{H}_{-}^{0}\left(\Gamma_{D}, \Gamma_{N}\right) \times \mathbb{C} \rightarrow \mathbb{C}^{p}:(f, g) \mapsto\left(u\left(Q_{1}\right), \ldots, u\left(Q_{p}\right)\right),
\end{gathered}
$$

where $u$ is defined as above by (3) and (4) respectively.

2.3. The mapping properties. If $\Gamma_{D}=\emptyset$, our ansatz (3) is the single layer potential. It defines a one to one boundary operator if and only if the capacity of $\Omega$ is not one, see for example 9 . In the general case, denote by $G$ the solution of the mixed problem

$$
\left\{\begin{array}{l}
\Delta G=0 \quad \text { in } \quad \mathbb{R}^{2} \backslash \bar{\Omega}, \\
G_{\mid \Gamma_{N}}=0, \quad \partial_{\nu} G_{\mid \Gamma_{D}}=0, \\
G(x)=\log |x|+O(1), \quad x \rightarrow+\infty .
\end{array}\right.
$$

The existence and some properties of this function are presented in Section 4. We define the mixed capacity $\gamma$ of $\Omega$ with respect to the decomposition $\left(\Gamma_{D}, \Gamma_{N}\right)$ of the boundary $\Gamma$ by the limit

$$
\log \gamma=\lim _{x \rightarrow \infty} G(x)-\log |x| .
$$

The central mapping properties of the boundary operator defined by (3) are summed up in the following result.

Theorem 1. If $s \geq 0$, then the operator $T_{ \pm}$maps $\mathcal{H}_{ \pm}^{s}\left(\Gamma_{D}, \Gamma_{N}\right)$ into $H_{c}^{s}\left(\Gamma_{D}\right) \times$ $H_{c}^{s}\left(\Gamma_{N}\right)$.

Assume that $s \geq 0$ and $s-\frac{1}{2} \notin e_{\omega_{j}}$ for every $j$. It follows that:

- If $\Gamma_{D} \neq \emptyset$ and the mixed capacity of $\Omega$ with respect to the decomposition $\left(\Gamma_{D}, \Gamma_{N}\right)$ is not 1 , then the operator

$\mathcal{T}_{+}: \mathcal{H}_{+}^{s}\left(\Gamma_{D}, \Gamma_{N}\right) \rightarrow H_{c}^{s}\left(\Gamma_{D}\right) \times H_{c}^{s}\left(\Gamma_{N}\right) \times \mathbb{C}^{p}:(f, g) \mapsto\left(T_{+}(f, g), S_{+}(f, g)\right)$

is bijective.

- If $\Gamma_{D} \neq \emptyset$, then the operator

$$
\begin{aligned}
\mathcal{T}_{-}: \mathcal{H}_{-}^{s}\left(\Gamma_{D}, \Gamma_{N}\right) \times \mathbb{C} & \rightarrow H_{c}^{s}\left(\Gamma_{D}\right) \times H_{c}^{s}\left(\Gamma_{N}\right) \times \mathbb{C}^{p+1}: \\
(f, g, c) & \mapsto\left(T_{-}(f, g), S_{-}(f, g), \int_{\Gamma_{N}} g d \sigma\right)
\end{aligned}
$$

is bijective. 
- If $\Gamma_{D}=\emptyset$, then $\mathcal{T}_{ \pm}$are Fredholm operators with index 0 and one dimensional kernel; $\mathcal{T}_{+}$has the same property if $\Gamma_{D} \neq \emptyset$ and the mixed capacity is 1.

The proof is given in Section 6

The condition on the capacity is analogous to the one met in the use of the single layer potential for the pure Dirichlet problem and should not be a real problem. Any dilation by a factor $r$ multiplies the capacity by $r$. Hence, for any open subset $\Omega$ of $\mathbb{R}^{2}$, there is one and only one $r>0$ such that $r \Omega$ has capacity 1 . Moreover, in this case, the addition of a good operator of rank one gives a bijective boundary operator. For example, if the mixed capacity is 1 , we can replace (3) by

$$
\begin{aligned}
K(g, h)(x)= & \frac{1}{2 \pi} \int_{\Gamma_{N}} g(y) \log |x-y| d \sigma(y) \\
& \quad+\frac{1}{2 \pi} \int_{\Gamma_{D}} \frac{(x-y) \cdot \nu_{y}}{|x-y|^{2}} h(y) d \sigma(y)+\frac{1}{2 \pi} \int_{\Gamma_{N}} g d \sigma .
\end{aligned}
$$

It follows from Proposition 6 that the corresponding boundary operator is bijective. To avoid lengthy discussions, we describe the numerical methods only in the cases where $\mathcal{T}_{+}$or $\mathcal{T}_{-}$is bijective. Hence, we assume in what follows that $\Gamma_{D} \neq \emptyset$.

2.4. The numerical methods. Let us describe the collocation methods. They are built using splines of odd degree. For simplicity, we only consider meshes that are uniform on each side. This is natural in our framework, since the singularities occurring near the corners are already taken into account by the method. We are looking for a Galerkin method which reduces to collocation equations, and adapt the trial and test spaces to the results obtained in Theorem 1

For any $j$ such that $0 \leq j<M$, let $n_{j}>0$ be the number of subdivisions of the sides $\Gamma_{j}$ and consider the points

$$
P_{j}=P_{j}^{(0)}=\gamma_{j}(0), P_{j}^{(1)}=\gamma_{j}\left(h_{j}\right), \ldots, P_{j}^{\left(n_{j}\right)}=\gamma_{j}\left(n_{j} h_{j}\right)=P_{j+1}
$$

with $h_{j}=t_{j} / n_{j}$. The set of these points is called a mesh $\Delta$ with meshwidth $\delta_{\Delta}=\sup _{j<M} h_{j}$.

Fix a strictly positive integer $m$ and a mesh $\Delta$. Assume that

$$
n_{j} \geq 2 m \text { and } 2 m-\frac{3}{2} \notin e_{\omega_{j}}
$$

for every $j$. Denote by $S_{\Delta, j, 0}^{(m)}$ the set of smoothest splines of degree $2 m-1$ on $\Gamma_{j}$ subordinated to the mesh $\Delta$ that vanish to order $2 m-1$ at the corners $P_{j}$ and $P_{j+1}$. Extend these functions by 0 on the other sides. The dimension of this space is $n_{j}-(2 m-1)$. Define

$$
S_{\Delta, 0}^{(m)}(\Gamma)=\sum_{j=0}^{M-1} S_{\Delta, j, 0}^{(m)}
$$

and

$$
V_{\Delta, \pm}^{(m)}(\Gamma)=S_{\Delta, 0}^{(m)}(\Gamma)+\sum_{j=0}^{M-1} \chi_{j} \mathcal{L}_{j, 2 m-1}^{ \pm},
$$

where the functions $\chi_{j}$ are chosen as in the definition of the spaces $\mathcal{H}^{s}\left(\Gamma_{D}, \Gamma_{N}\right)$. 
Denote by $V_{\Delta, v, \pm}^{(m)}(\Gamma)$ the subspace of $V_{\Delta, \pm}^{(m)}(\Gamma)$ formed by the elements whose integral on each connected component of $\Gamma_{D}$ is 0 . By construction, we have

$$
V_{\Delta, v, \pm}^{(m)}(\Gamma) \subset \mathcal{H}_{ \pm}^{2 m-1}\left(\Gamma_{D}, \Gamma_{N}\right)
$$

and

$$
\begin{aligned}
\operatorname{dim} V_{\Delta, v, \pm}^{(m)}(\Gamma) & =\sum_{j=0}^{M-1}\left(n_{j}-(2 m-1)+2(2 m-1)\right)+p \\
& =\sum_{j=0}^{M-1} n_{j}+M(2 m-1)+p
\end{aligned}
$$

Indeed, in a nonmixed corner we have $2(2 m-1)$ singular functions. For each component $\Gamma_{D, j}$ of $\Gamma_{D}$, we have to add the two additional singular functions generated by the mixed corners, and subtract one because the integral of the boundary unknown has to vanish on $\Gamma_{D, j}$.

Our space of trial functions for (3) or (4) is the space $W_{\Delta, \pm}^{(m)}(\Gamma)$ of functions

$$
(g, h) \in \widetilde{H}^{-1 / 2}\left(\Gamma_{N}\right) \times \widetilde{H}^{1 / 2}\left(\Gamma_{D}\right)
$$

such that $\left(\partial_{t} h, g\right) \in V_{\Delta, v, \pm}^{(m)}(\Gamma)$. This means that we take an approximation of $g$ in the linear hull of the singular functions and of the splines of order $2 m-1$, whereas we use the linear hull of the antiderivatives of the same singular functions and of the splines of order $2 m$ for the approximation of $h$. This shift of regularity corresponds exactly to the shift of spaces in the variational setting.

Assume that $u_{0} \in H^{m+1}\left(\Gamma_{D}\right)$ and $u_{1} \in H_{c}^{m}\left(\Gamma_{N}\right)$, and denote by $v$ the function equal to $\partial_{t} u_{0}$ on $\Gamma_{D}$ and $u_{1}$ on $\Gamma_{N}$. If $f$ is a smooth function on $\Gamma_{j}$, denote by $\partial_{+, j}^{k} f$ (resp. $\left.\partial_{-, j}^{k} f\right)$ the derivative of order $k$ of $f$ at $P_{j}$ (resp. $P_{j+1}$ ) in the direction of the unit tangent vector of $\Gamma_{j}$. To solve (1), we consider the following collocation equations.

Find $(g, h) \in W_{\Delta,+}^{(m)}(\Gamma)$ such that

$$
\left\{\begin{array}{lll}
\partial_{t} K(g, h)\left(P_{j}^{(k)}\right)=v\left(P_{j}^{(k)}\right), & j \in e_{D}, & 0 \leq k \leq n_{j}, \\
\partial_{\nu} K(g, h)\left(P_{j}^{(k)}\right)=v\left(P_{j}^{(k)}\right), & j \in e_{N}, & 0 \leq k \leq n_{j}, \\
K(g, h)\left(Q_{j}\right)=u_{0}\left(Q_{j}\right), & 1 \leq j \leq p, & \\
\partial_{ \pm, j}^{\ell+1} K(g, h)=\partial_{ \pm, j}^{\ell} v, & j \in e_{D}, & 0<\ell<m, \\
\partial_{ \pm, j}^{\ell} \partial_{\nu} K(g, h)=\partial_{ \pm, j}^{\ell} v, & j \in e_{N}, & 0<\ell<m .
\end{array}\right.
$$

By construction, the number of equalities in (6) is equal to the dimension of $W_{\Delta, \pm}^{(m)}(\Gamma)$. The derivatives that are used at the corners are exactly the ones that make sense for the boundary values of $K(g, h)$ in view of Theorem 1 if $u_{0} \in$ $H^{m+1}\left(\Gamma_{D}\right)$ and $u_{1} \in H_{c}^{m}\left(\Gamma_{N}\right)$.

As explained in Section [6 and 7 (see (13), (14) and also (16), (17)), all the derivatives that occur in these collocation equations can be performed analytically on the formula giving $K$ and hence can be easily computed.

To solve (2), we use essentially the same method. Let $a \in \mathbb{C}, u_{0} \in H^{m+1}\left(\Gamma_{D}\right)$, $u_{1} \in H_{c}^{m}\left(\Gamma_{N}\right)$, and denote as above by $v$ the function equal to $\partial_{t} u_{0}$ on $\Gamma_{D}$ and $u_{1}$ on $\Gamma_{N}$. We consider the solution $(g, h, c) \in W_{\Delta,-}^{(m)}(\Gamma) \times \mathbb{C}$ of the collocation 
equations

$$
\left\{\begin{array}{lll}
\partial_{t} K(g, h, c)\left(P_{j}^{(k)}\right)=v\left(P_{j}^{(k)}\right), & j \in e_{D}, & 0 \leq k \leq n_{j}, \\
\partial_{\nu} K(g, h, c)\left(P_{j}^{(k)}\right)=v\left(P_{j}^{(k)}\right), & j \in e_{N}, & 0 \leq k \leq n_{j}, \\
K(g, h, c)\left(Q_{j}\right)=u_{0}\left(Q_{j}\right), & 1 \leq j \leq p, & \\
\partial_{ \pm, j}^{\ell+1} K(g, h, c)=\partial_{ \pm, j}^{\ell} v, & j \in e_{D}, & 0<\ell<m, \\
\partial_{ \pm, j}^{\ell} \partial_{\nu} K(g, h, c)=\partial_{ \pm, j}^{\ell} v, & j \in e_{N}, & 0<\ell<m, \\
\frac{1}{2 \pi} \int_{\Gamma_{N}} g d \sigma=a . & &
\end{array}\right.
$$

Here again, we get a square linear system.

Our main convergence result is the following quasi-optimal estimate.

Theorem 2. Let $\Omega$ be a bounded polygonal open subset of $\mathbb{R}^{2}$ and $\left(\Gamma_{D}, \Gamma_{N}\right)$ a decomposition of the boundary defining a mixed capacity $\gamma \neq 1$. If $m$ is a strictly positive integer such that $m-\frac{1}{2} \notin e_{\omega_{j}}$ and $2 m-\frac{3}{2} \notin e_{\omega_{j}}$ for every $j$, then there are $C, \delta>0$ such that, for every mesh $\Delta$ with meshwidth $\delta_{\Delta}$ less than $\delta$ and every functions $\left(u_{0}, u_{1}\right) \in H^{m+1}\left(\Gamma_{D}\right) \times H^{m}\left(\Gamma_{N}\right)$, the solution $\left(g_{\Delta}, h_{\Delta}\right)$ of the collocation equations (6) is unique and satisfies

$$
\begin{aligned}
\|\left(\partial_{t} h, g\right) & -\left(\partial_{t} h_{\Delta}, g_{\Delta}\right) \|_{\mathcal{H}_{+}^{m}\left(\Gamma_{D}, \Gamma_{N}\right)} \\
\leq & C \inf _{(\tilde{g}, \tilde{h}) \in W_{\Delta,+}^{(m)}(\Gamma)}\left\|\left(\partial_{t} h, g\right)-\left(\partial_{t} \tilde{h}, \tilde{g}\right)\right\|_{\mathcal{H}_{+}^{m}\left(\Gamma_{D}, \Gamma_{N}\right)},
\end{aligned}
$$

where $(g, h) \in \widetilde{H}^{-1 / 2}\left(\Gamma_{N}\right) \times \widetilde{H}^{1 / 2}\left(\Gamma_{D}\right)$ satisfies $\left(\partial_{t} h, g\right) \in \mathcal{H}_{+}^{m}\left(\Gamma_{D}, \Gamma_{N}\right)$ and is the solution of

$$
K(g, h)_{\mid \Gamma_{D}}=u_{0}, \quad \partial_{\nu} K(g, h)_{\mid \Gamma_{N}}=u_{1} .
$$

The same result holds for the exterior problem with data

$$
\left(u_{0}, u_{1}, a\right) \in H^{m+1}\left(\Gamma_{D}\right) \times H^{m}\left(\Gamma_{N}\right) \times \mathbb{C}
$$

and approximation $\left(g_{\Delta}, h_{\Delta}, c_{\Delta}\right) \in W_{\Delta--}^{(m)}(\Gamma) \times \mathbb{C}$.

As a consequence of this result and of the approximation properties of splines, we obtain the following orders of convergence. We state it for the interior problem.

Theorem 3. Under the conditions of Theorem Q, there are constants $C(s)>0$ such that

$$
\left\|\left(\partial_{t} h, g\right)-\left(\partial_{t} h_{\Delta}, g_{\Delta}\right)\right\|_{\mathcal{H}_{+}^{k}\left(\Gamma_{D}, \Gamma_{N}\right)} \leq C(s) \delta_{\Delta}^{s-k}\left\|\left(u_{0}, u_{1}\right)\right\|_{H^{s+1}\left(\Gamma_{D}\right) \times H^{s}\left(\Gamma_{N}\right)}
$$

if $k$ is an integer, $k-\frac{1}{2} \notin e_{\omega_{j}}$ for every $j, 0 \leq k \leq m \leq s<\alpha+\frac{1}{2}$, where $\alpha$ is the smallest element of $\bigcup_{j=0}^{N-1} e_{\omega_{j}}$ such that $\alpha>2 m-\frac{3}{2}, s-\frac{1}{2} \notin e_{\omega_{j}}$ for every $j$ and $\left(u_{0}, u_{1}\right) \in H^{s+1}\left(\Gamma_{D}\right) \times H^{s}\left(\Gamma_{N}\right)$.

The exact value of the first missed singular exponent depends on the polygon. However, we always have $2 m-\frac{3}{2}<\alpha \leq 2 m-1$.

Theorems 2 and 3 are proved in Section $[7$ 


\section{THE VARIATIONAL FRAMEWORK}

We fix the general functional framework of our problem and give some references for the convenience of the reader. The notations are the same as in Section [2,

Denote by $\bar{C}^{\infty}(\Omega)$ the set of restrictions to $\Omega$ of the $C^{\infty}$ functions in $\mathbb{R}^{2}$, and let

$$
E\left(\Delta, L^{2}(\Omega)\right)=\left\{u \in H^{1}(\Omega): \Delta u \in L^{2}(\Omega)\right\}
$$

be the maximal domain for the Laplacian. Any element $u \in E\left(\Delta, L^{2}(\Omega)\right)$ has a boundary value $\gamma_{0} u \in H^{1 / 2}(\Gamma)$. The set $\bar{C}^{\infty}(\Omega)$ is dense $E\left(\Delta, L^{p}(\Omega)\right)$, and the trace map

$$
\gamma_{1} u=\left(\partial_{\nu} u\right)_{\mid \partial \Omega}
$$

defined on $\bar{C}^{\infty}(\Omega)$ extends as a continuous linear operator from $E\left(\Delta, L^{2}(\Omega)\right)$ into $H^{-1 / 2}(\partial \Omega)$. Moreover, if $u \in E\left(\Delta, L^{2}(\Omega)\right)$ and $v \in H^{1}(\Omega)$, we have

$$
\int_{\Omega} \Delta u \bar{v} d \lambda+\int_{\Omega} \sum_{j=1}^{2} D_{x_{j}} u \overline{D_{x_{j}} v} d \lambda=-\left\langle\gamma_{1} u, \gamma_{0} v\right\rangle .
$$

These results are proved in [10] or 6] for any bounded Lipschitz domain. It follows that, if $u \in H^{1}(\Omega)$ and $\Delta u=0$, then $u$ defines an element $\gamma_{1} u \in H^{-1 / 2}(\Gamma)$, hence an element of $H^{-1 / 2}\left(\Gamma_{N}\right)$.

If $\Gamma_{D}$ is not empty, $u_{0} \in H^{1 / 2}\left(\Gamma_{D}\right)$ and $u_{1} \in H^{-1 / 2}\left(\Gamma_{N}\right)$, then there is one and only one $u \in H^{1}(\Omega)$ such that $\gamma_{0} u_{\mid \Gamma_{D}}=u_{0}$ and

$$
\int_{\Omega} \operatorname{grad} u \cdot \overline{\operatorname{grad} v} d \lambda=u_{1}\left(\gamma_{0} v_{\mid \Gamma_{N}}\right)
$$

for every $v \in H^{1}(\Omega)$ satisfying $\gamma_{0} v_{\mid \Gamma_{D}}=0$. If $\Gamma_{D}$ is empty and $u_{1} \in H^{-1 / 2}(\Gamma)$ satisfies

$$
\int_{\Gamma} u_{1} d \sigma=0
$$

then there is a unique solution to the same problem in $H^{1}(\Omega) / \mathbb{R}$. This is the variational solution of the mixed problem

$$
\left\{\begin{array}{l}
\Delta u=0 \text { in } \Omega \\
u_{\mid \Gamma_{D}}=u_{0}, \quad \partial_{\nu} u_{\mid \Gamma_{N}}=u_{1} .
\end{array}\right.
$$

The exterior problem is solved in the same way.

Let us consider the boundary potentials. It follows from Theorem 1 of 2 that if $h \in \widetilde{H}^{1 / 2}\left(\Gamma_{D}\right)$ and $g \in \widetilde{H}^{-1 / 2}\left(\Gamma_{N}\right)$, then the function $u$ defined by (3) belongs to $E\left(\Delta, L^{2}(\Omega)\right)$. Hence, it has a boundary value $\gamma_{0} u \in H^{1 / 2}(\Gamma)$ and a normal derivative $\gamma_{1} u=\partial_{\nu} u \in H^{-1 / 2}(\Gamma)$. However, the functions $g$ and $h$ do not belong to the same spaces and the transition leads to problems in the collocation methods. We shift to other spaces.

As above, we define $\widetilde{H}^{-1 / 2}(] 0,1[)$ as the dual of $H^{1 / 2}(] 0,1[)$ and $H^{-1 / 2}(] 0,1[)$ as the dual of $\widetilde{H}^{1 / 2}(] 0,1[)$. It is known, [10] pp. 31-32, that the derivative defines a continuous operator from $H^{1 / 2}(] 0,1[)$ into $H^{-1 / 2}(] 0,1[)$ but not into $\widetilde{H}^{-1 / 2}(] 0,1[)$. We also have the following dual result. We give a proof for the sake of completeness.

Lemma 4. The derivative $D$ defines a bijective continuous operator from the space $\widetilde{H}^{1 / 2}(] 0,1[)$ onto the subspace of $\widetilde{H}^{-1 / 2}(] 0,1[)$ formed by the elements whose integral is 0 . 
Proof. Let $f \in \widetilde{H}^{1 / 2}(] 0,1[)$. By definition, the zero extension $\widetilde{f}$ belongs to $H^{1 / 2}(\mathbb{R})$. Hence $g=D \widetilde{f} \in H^{-1 / 2}(\mathbb{R})$. If $\varphi \in H^{1 / 2}(] 0,1[)$, then $h(\varphi)=g(\widetilde{\varphi})$ is independent of the extension $\widetilde{\varphi}$ of $\varphi$ as an element of $H^{1 / 2}(\mathbb{R})$. Indeed, $g$ vanishes on $C_{0}^{\infty}(\mathbb{R} \backslash[0,1])$ since its support is included in $[0,1]$. Moreover the difference of two extensions belongs to $\widetilde{H}^{1 / 2}(\mathbb{R} \backslash[0,1])$ and $C_{0}^{\infty}(\mathbb{R} \backslash[0,1])$ is dense in this space, [10], p. 24 . Since there is a continuous linear extension operator from $H^{1 / 2}(] 0,1[)$ to $H^{1 / 2}(\mathbb{R})$, $h$ belongs to $\widetilde{H}^{-1 / 2}(] 0,1[)$. It coincides with $g$ in $] 0,1[$. Hence

$$
D f=g_{\mid] 0,1[}=h \in \widetilde{H}^{-1 / 2}(] 0,1[) .
$$

Moreover, if $\chi \in C_{0}^{\infty}(\mathbb{R})$ is equal to 1 near $[0,1]$, we have

$$
\langle D f, 1\rangle=\langle h, 1\rangle=\langle g, \chi\rangle=\langle D \widetilde{f}, \chi\rangle=-\langle\widetilde{f}, D \chi\rangle=0 .
$$

The operator is one to one since the nonvanishing constants do not belong to $\widetilde{H}^{1 / 2}(] 0,1[)$.

Let us prove that it is onto. Let $f \in \widetilde{H}^{-1 / 2}(] 0,1[)$ be such that $\langle f, 1\rangle=0$. Define $u=H^{-1 / 2}(\mathbb{R})$ by

$$
u(\varphi)=f\left(\varphi_{\mid] 0,1[}\right), \quad \varphi \in H^{1 / 2}(\mathbb{R}) .
$$

The Fourier transform $\widehat{u}$ of $u$ is an entire function vanishing at 0 . By the PaleyWiener theorem, there is $v \in H^{1 / 2}(\mathbb{R})$ such that $\widehat{v}(\xi)=\widehat{u}(\xi) / i \xi$ and $\operatorname{supp} v \subset[0,1]$. The restriction of $v$ to $] 0,1\left[\right.$ belongs to $\widetilde{H}^{1 / 2}(] 0,1[)$, and its derivative is $f$.

Let $\sigma_{j}:\left[0, L_{j}\right] \rightarrow \Gamma_{D, j}$ be the parameterization by arc length of the $j$-th connected component of $\Gamma_{D}$. For each $j$, the space $H^{1 / 2}\left(\Gamma_{D, j}\right)$ is formed by the functions $u$ such that $u \circ \sigma_{j} \in H^{1 / 2}(] 0, L_{j}[)$. Hence, as a corollary of Lemma 4 we obtain the following result.

Corollary 5. The derivative with respect to the unit tangent vector maps the space $\widetilde{H}^{1 / 2}\left(\Gamma_{D}\right)$ onto the space $\widetilde{H}_{v}^{-1 / 2}\left(\Gamma_{D}\right)$.

\section{THE MIXED CAPACITY}

As in Section 2, let $\Omega$ be a bounded polygonal open subset of $\mathbb{R}^{2}$. We fix a partition $\Gamma=\Gamma_{D} \cup \Gamma_{N}$ of its boundary.We assume that $\Gamma_{N} \neq \emptyset$. There is a unique harmonic function in $\mathbb{R}^{2} \backslash \bar{\Omega}$ such that

$$
G \in H^{1}\left(\left(\mathbb{R}^{2} \backslash \bar{\Omega}\right) \cap\left\{x \in \mathbb{R}^{2}:|x|<R\right\}\right)
$$

for any $R>0$ and

$$
\left\{\begin{array}{l}
\Delta G=0 \quad \text { in } \quad \mathbb{R}^{2} \backslash \bar{\Omega}, \\
G_{\mid \Gamma_{N}}=0, \quad \partial_{\nu} G_{\mid \Gamma_{D}}=0, \\
G(x)=\log |x|+O(1), \quad x \rightarrow+\infty .
\end{array}\right.
$$

This is the Green function $G$ with pole at infinity. By a classical theorem of Riemann, the function $G(x)-\log |x|$ is harmonic at infinity. We define the mixed capacity $\gamma$ of $\Omega$ with respect to the decomposition $\left(\Gamma_{D}, \Gamma_{N}\right)$ by the limit

$$
\log \gamma=\lim _{x \rightarrow \infty} G(x)-\log |x| .
$$


Proposition 6. Let $G$ be the Green function of $\Omega$ associated to a decomposition $\left(\Gamma_{D}, \Gamma_{N}\right)$ of the boundary of $\Omega$, and let $\gamma$ be the mixed capacity of $\Omega$. If $x \in \mathbb{R}^{2} \backslash \bar{\Omega}$, then

$$
G(x)=\log \gamma-\frac{1}{2 \pi} \int_{\Gamma_{D}} \frac{(x-y) \cdot \nu_{y}}{|x-y|^{2}} G(y) d \sigma(y)-\frac{1}{2 \pi} \int_{\Gamma_{N}} \partial_{\nu} G(y) \log |x-y| d \sigma(y) .
$$

If $x \in \Omega$, then

$$
\frac{1}{2 \pi} \int_{\Gamma_{D}} \frac{(x-y) \cdot \nu_{y}}{|x-y|^{2}} G(y) d \sigma(y)+\frac{1}{2 \pi} \int_{\Gamma_{N}} \partial_{\nu} G(y) \log |x-y| d \sigma(y)=\log \gamma .
$$

Moreover, if $g \in \widetilde{H}^{-1 / 2}\left(\Gamma_{N}\right), h \in \widetilde{H}^{1 / 2}\left(\Gamma_{D}\right)$ and

$$
\frac{1}{2 \pi} \int_{\Gamma_{N}} g(y) \log |x-y| d \sigma(y)+\frac{1}{2 \pi} \int_{\Gamma_{D}} \frac{(x-y) \cdot \nu_{y}}{|x-y|^{2}} h(y) d \sigma(y)
$$

is constant in $\Omega$, then

$$
g=-\frac{\partial_{\nu} G}{2 \pi} \int_{\Gamma_{N}} g d \sigma, \quad h=-\frac{G}{2 \pi} \int_{\Gamma_{N}} g d \sigma .
$$

Proof. Assume that $\bar{\Omega}$ is included in the open ball with center 0 and radius $R$. If $x \in \mathbb{R}^{2} \backslash \bar{\Omega}$ and $|x|<R$, the Green formula gives

$$
\begin{aligned}
G(x)= & \frac{1}{2 \pi} \int_{|y|=R} \frac{(x-y) \cdot \nu_{y}}{|x-y|^{2}} G(y) d \sigma(y)+\frac{1}{2 \pi} \int_{|y|=R} \partial_{\nu} G(y) \log |x-y| d \sigma(y) \\
& -\frac{1}{2 \pi} \int_{\Gamma_{D}} \frac{(x-y) \cdot \nu_{y}}{|x-y|^{2}} G(y) d \sigma(y)-\frac{1}{2 \pi} \int_{\Gamma_{N}} \partial_{\nu} G(y) \log |x-y| d \sigma(y)
\end{aligned}
$$

with $\nu_{y}=-y /|y|$ on $|y|=R$. By construction of $G$, we have

$$
G(x)=\log r+\log \gamma+O\left(\frac{1}{r}\right), \quad \partial_{r} G(x)=\frac{1}{r}+O\left(\frac{1}{r^{2}}\right)
$$

with $r=|x|$. Using this asymptotic behavior in the first integrals and considering the limit for $R \rightarrow+\infty$, we get

$$
G(x)=\log \gamma-\frac{1}{2 \pi} \int_{\Gamma_{D}} \frac{(x-y) \cdot \nu_{y}}{|x-y|^{2}} G(y) d \sigma(y)-\frac{1}{2 \pi} \int_{\Gamma_{N}} \partial_{\nu} G(y) \log |x-y| d \sigma(y)
$$

in $\mathbb{R}^{2} \backslash \bar{\Omega}$. The right hand side defines a harmonic function in $\Omega$. On $\Gamma_{D}$, its normal derivative vanishes since the normal derivative of the double layer potential is continuous across $\Gamma_{D}$; see Lemma 4.1 of 2 . On $\Gamma_{N}$, it vanishes since the single layer potential is continuous across $\Gamma_{N}$. The mixed problem in $\Omega$ has a unique solution hence the right hand side vanishes in $\Omega$.

Assume that $g \in \widetilde{H}^{-1 / 2}\left(\Gamma_{N}\right), h \in \widetilde{H}^{1 / 2}\left(\Gamma_{D}\right)$ and that the function

$u(x)=\frac{1}{2 \pi} \int_{\Gamma_{N}} g(y) \log |x-y| d \sigma(y)+\frac{1}{2 \pi} \int_{\Gamma_{D}} \frac{(x-y) \cdot \nu_{y}}{|x-y|^{2}} h(y) d \sigma(y), \quad x \in \mathbb{R}^{2} \backslash \partial \Omega$,

is equal to a constant $c$ in $\Omega$. If $x \in \mathbb{R}^{2} \backslash \bar{\Omega}$, let

By construction

$$
v(x)=u(x)-\frac{G(x)}{2 \pi} \int_{\Gamma_{N}} g d \sigma .
$$

$$
v(x)=-\frac{\log \gamma}{2 \pi} \int_{\Gamma_{N}} g d \sigma+O\left(\frac{1}{r}\right) .
$$


Using the Green formula (18), we obtain

$$
\int_{\mathbb{R}^{2} \backslash \bar{\Omega}}|\operatorname{grad} v|^{2} d \lambda=\left\langle\partial_{\nu} v_{\mid \Gamma}, v_{\mid \Gamma}-c\right\rangle .
$$

This expression is 0 . Indeed, on $\Gamma_{N}$ we have $u-c=G=0$, since the single layer potential is continuous across the boundary. On $\Gamma_{D}$, we have $\partial_{\nu} u=\partial_{\nu} G=0$, since the normal derivative of the double layer potential is continuous across the boundary. It follows that $v$ is a constant. Since it is equal to $c$ on $\Gamma_{N}$, we obtain

$$
c=-\frac{\log \gamma}{2 \pi} \int_{\Gamma_{N}} g d \sigma .
$$

It follows that

$$
u(x)= \begin{cases}c & \text { in } \quad \Omega, \\ c+\frac{G(x)}{2 \pi} \int_{\Gamma_{N}} g d \sigma & \text { in } \quad \mathbb{R}^{2} \backslash \partial \Omega .\end{cases}
$$

Computing the jump on $\Gamma_{D}$ and the jump of the normal derivative on $\Gamma_{N}$ of this expression of $u$, we obtain

$$
g=-\frac{\partial_{\nu} G}{2 \pi} \int_{\Gamma_{N}} g d \sigma, \quad h=-\frac{G}{2 \pi} \int_{\Gamma_{N}} g d \sigma
$$

This proves the proposition.

\section{Mellin analysis in a CORner}

We are looking for a bijective boundary operator between spaces of high regularity. This will be a consequence of the previous global properties of the potential as well as of local properties near each corner. In this section, we perform a Mellin analysis to obtain the singularities generated by these corners and to prove the coerciveness of the boundary operator in suitable spaces.

5.1. Mixed corner. Let us consider the unbounded corner

$$
\Omega=\left\{r e^{i \theta}: r>0,0<\theta<\omega\right\}
$$

with $0<\omega<2 \pi$ and $\omega \neq \pi$. Here again $\nu$ denotes the unit inward normal vector. Assume that $\Gamma_{D}$ is the real axis and $\Gamma_{N}$ the other part of the boundary.

Let $(g, h) \in \widetilde{H}^{-1 / 2}\left(\mathbb{R}_{+}\right) \times \widetilde{H}^{1 / 2}\left(\mathbb{R}_{+}\right)$and $u=K(g, h)$ with the notations of (3). Its boundary values are given by

$$
\left(\begin{array}{c}
u_{\mid \Gamma_{D}} \\
\partial_{\nu} u_{\mid \Gamma_{N}}
\end{array}\right)=\frac{1}{2}\left(\begin{array}{cc}
I & V \\
S & I
\end{array}\right)\left(\begin{array}{c}
h \\
g
\end{array}\right)
$$

with

$$
V g(x)=\frac{1}{\pi} \int_{0}^{+\infty} g(y) \log \sqrt{x^{2}+y^{2}-2 x y \cos \omega} d y
$$

and

$$
S h(x)=\frac{1}{\pi} \int_{0}^{+\infty} \frac{2 x y-\left(x^{2}+y^{2}\right) \cos \omega}{\left(x^{2}+y^{2}-2 x y \cos \omega\right)^{2}} h(y) d y .
$$

Using the direct method, a similar operator is obtained in [3]. It is strongly elliptic in the energy norm. However, it is not even continuous in the natural space for the Galerkin procedure. This problem is partially avoided in [3] by using a Gaussian type elimination near the mixed corners. Here, we perform collocation on the 
tangential derivative of the Dirichlet data and on the normal derivative of the solution on the Neumann part of the boundary. Moreover, we use $f=D h$ and $g$ as unknown. This leads to a strongly elliptic operator in high order Sobolev spaces.

If $x>0$, we get

$$
D(V g)(x)=\frac{1}{\pi} \int_{0}^{+\infty} \frac{x-y \cos \omega}{x^{2}+y^{2}-2 x y \cos \omega} g(y) d y .
$$

On the other hand,

$$
\frac{2 x y-\left(x^{2}+y^{2}\right) \cos \omega}{\left(x^{2}+y^{2}-2 x y \cos \omega\right)^{2}}=-D_{y}\left(\frac{x-y \cos \omega}{x^{2}+y^{2}-2 x y \cos \omega}\right) .
$$

It follows that

$$
\operatorname{Sh}(x)=\frac{1}{\pi} \int_{0}^{+\infty} \frac{x-y \cos \omega}{x^{2}+y^{2}-2 x y \cos \omega} D h(y) d y,
$$

since this is true for $h \in C_{0}^{\infty}\left(\mathbb{R}_{+}\right)$and this space is dense in $\widetilde{H}^{1 / 2}\left(\mathbb{R}_{+}\right)$. Of course, in the general case the integrals are taken in the sense of duality.

This means that

$$
\mathcal{W}_{\omega}\left(\begin{array}{c}
f \\
g
\end{array}\right):=T_{+}(f, g)=\left(\begin{array}{c}
\partial_{t} u_{\mid \Gamma_{D}} \\
\partial_{\nu} u_{\mid \Gamma_{N}}
\end{array}\right)=\frac{1}{2}\left(\begin{array}{cc}
I & W \\
W & I
\end{array}\right)\left(\begin{array}{l}
f \\
g
\end{array}\right)
$$

with

$$
W f(x)=\frac{1}{\pi} \int_{0}^{+\infty} \frac{x-y \cos \omega}{x^{2}+y^{2}-2 x y \cos \omega} f(y) d y .
$$

If $\Gamma_{N}$ is the real axis and $\Gamma_{D}$ the other part of the boundary, we have to replace $W$ by $-W$. If we consider the exterior case, we have to replace $I$ by $-I$. Since these modifications are only sign changes and the definition of $\mathcal{L}_{j, s}^{ \pm}$takes care of this, we can restrict ourselves to $\mathcal{W}_{\omega}$ in the next result.

Proposition 7. If $\omega \in] 0,2 \pi\left[\backslash\{\pi\}, s \geq 0\right.$ and $\chi \in C_{0}^{\infty}(\mathbb{R})$ is equal to 1 in a neighborhood of 0 , then the operator

$$
\mathcal{W}_{\omega}: H_{0}^{s}\left(\mathbb{R}_{+}\right)^{2}+\chi \mathcal{L}_{\omega, s,+}^{(m)} \rightarrow H^{s}\left(\mathbb{R}_{+}\right)^{2}
$$

is continuous. Moreover, if $s-\frac{1}{2} \notin e_{\omega}^{(m)}$, it is onto and its kernel is the linear hull of the function $\left(x^{\alpha},-x^{\alpha}\right)$, where $\alpha$ is the element of $e_{\omega}^{(m)}$ satisfying $-1<\alpha<-1 / 2$.

If $m$ is an integer and $m-\frac{1}{2} \notin e_{\omega}^{(m)}$, then there is $c>0$ such that

$$
\left\langle D^{m} \mathcal{W}_{\omega} v, D^{m} v\right\rangle_{L^{2}\left(\mathbb{R}_{+}\right)^{2}} \geq c\left\|D^{m} v\right\|_{L^{2}\left(\mathbb{R}_{+}\right)^{2}}^{2}
$$

for any $v \in H_{0}^{m}\left(\mathbb{R}_{+}\right)^{2}$.

As usual in the Fix method, we have to avoid that a singular function lies exactly at the boundary of the Sobolev regularity under consideration. This should not be a real restriction in practical computations.

Proof. From a classical lemma of Hilbert, see [11, p. 229, it follows that $W$ is continuous from $L^{2}\left(\mathbb{R}_{+}\right)$into itself. To obtain more precise results, we use the Mellin transform

$$
M f(z)=\int_{0}^{+\infty} x^{z-1} f(x) d x, \quad f \in C_{0}^{\infty}\left(\mathbb{R}_{+}\right) .
$$


The operator mapping $f$ to $t \mapsto M f\left(\frac{1}{2}+i t\right)$ extends as an isomorphism from $L^{2}\left(\mathbb{R}_{+}\right)$ onto $L^{2}(\mathbb{R})$. Indeed, this is the composition of the operator mapping $f \in L^{2}\left(\mathbb{R}_{+}\right)$ to $s \mapsto e^{-s / 2} f\left(e^{-s}\right)$ and the Fourier transform in $L^{2}(\mathbb{R})$. Let us prove that if $f \in L^{2}\left(\mathbb{R}_{+}\right)$and $\Re z=\frac{1}{2}$, we have

$$
M(W f)(z)=\frac{\cos ((z-1)(\pi-\omega))}{\sin (\pi z)} M f(z) .
$$

By density, we can assume that $f \in C_{0}^{\infty}\left(\mathbb{R}_{+}\right)$. In this case, we can write

$$
\begin{aligned}
M(W f)(z) & =\frac{1}{\pi} \int_{0}^{+\infty} x^{z-1} d x \int_{0}^{+\infty} \frac{x-y \cos \omega}{x^{2}+y^{2}-2 x y \cos \omega} f(y) d y \\
& =\frac{1}{\pi} \int_{0}^{+\infty} f(y) d y \int_{0}^{+\infty} \frac{x^{z-1}(x-y \cos \omega)}{x^{2}+y^{2}-2 x y \cos \omega} d x .
\end{aligned}
$$

Using the method of residues to compute the integrals of Euler type, see for example [1] p. 157, we get

$$
\begin{aligned}
\int_{0}^{+\infty} \frac{x^{z-1}(x-y \cos \omega)}{x^{2}+y^{2}-2 x y \cos \omega} d x & =i \pi y^{z-1} \frac{e^{i \omega(z-1)}+e^{i(2 \pi-\omega)(z-1)}}{1-e^{2 i \pi z}} \\
& =\pi y^{z-1} \frac{\cos ((z-1)(\pi-\omega))}{\sin (\pi z)}
\end{aligned}
$$

if $0<\Re z<1$ and $y>0$. This proves the required formula.

This shows that, on the Mellin side, the operator $\mathcal{W}_{\omega}$ is the multiplication by

$$
S_{\omega}(z)=\left(\begin{array}{cc}
1 & \frac{\cos ((z-1)(\pi-\omega))}{\sin (\pi z)} \\
\frac{\cos ((z-1)(\pi-\omega))}{\sin (\pi z)} & 1
\end{array}\right) .
$$

Since $\omega \neq \pi$, this matrix is invertible on the line $\Re z=\frac{1}{2}$ and has a bounded inverse. Hence $\mathcal{W}_{\omega}$ is an isomorphism from $L^{2}$ onto itself.

Let us prove the mapping properties in $H_{0}^{s}\left(\mathbb{R}_{+}\right)^{2}+\chi \mathcal{L}_{\omega, s,+}^{(m)}$. By construction, the elements of $e_{\omega}^{(m)}$ are the real numbers $\alpha>-1$ such that $z=-\alpha$ is a root of

$$
\cos ((z-1)(\pi-\omega))^{2}-\sin (\pi z)^{2}=0 .
$$

All these roots are real and $1 / 2$ is never a root. Such a root is double if and only if it can be written $z=\ell / 2$ with $\ell \in \mathbb{Z}$. It never has the multiplicity 3 .

We first remark that the function $\left(x^{\alpha},-x^{\alpha}\right)$ is in the kernel of $\mathcal{W}_{\omega}$ if $-1<\alpha<$ $-1 / 2$ and $\alpha \in e_{\omega}^{(m)}$. Indeed, using again the method of residues, we get in this case

$$
\frac{1}{\pi} \int_{0}^{+\infty} \frac{(x-y \cos \omega) y^{\alpha}}{x^{2}+y^{2}-2 x y \cos \omega} d y=-x^{\alpha} \frac{\cos (\alpha+1)(\pi-\omega)}{\sin (\pi \alpha)}=x^{\alpha} .
$$

If $\alpha>-1 / 2$, the previous argument cannot be used since $x^{\alpha}$ does not belong to $L^{2}$ at infinity. We use the characterization of the Sobolev spaces $H^{s}\left(\mathbb{R}_{+}\right)$by the Mellin transform (see Theorem 4 in [14] or Theorem 1.1.23 in [17]) and the following fact. The Mellin transform of $x^{\alpha} \chi(x)$ extends as a meromorphic function in $\mathbb{C}$ with a simple pole at $-\alpha$. Moreover, for every $M>0$, there are constants $C_{k}>0$ such that the estimation

$$
\left|M\left(x^{\alpha} \chi\right)(z)\right| \leq \frac{C_{k}}{(1+|\Im z|)^{k}} \frac{1}{|z+\alpha|}
$$


holds if $|\Re z| \leq M$. This follows from the equality

$$
\begin{gathered}
(z+\alpha)(z+\alpha+1) \ldots(z+\alpha+k) \int_{0}^{+\infty} x^{z+\alpha-1} \chi(x) d x \\
=\int_{0}^{+\infty} x^{z+\alpha+k}(-D)^{k+1} \chi(x) d x .
\end{gathered}
$$

Since $D^{k+1} \chi$ vanishes near 0 if $k \geq 0$, the right hand side is bounded in any vertical strip of the complex plane. In the same way

$$
\begin{aligned}
(z+\alpha) & \int_{0}^{+\infty} x^{z+\alpha-1} \log (x) \chi(x) d x \\
& =-\int_{0}^{+\infty} x^{z+\alpha-1} \chi(x) d x-\int_{0}^{+\infty} x^{z+\alpha} \log (x) D \chi(x) d x .
\end{aligned}
$$

Hence, for the function $x^{\alpha} \log (x) \chi(x)$, we have a pole of order 2 at $-\alpha$ and the same estimation with $|z+\alpha|$ replaced by $|z+\alpha|^{2}$.

Let $\widetilde{\mathcal{L}}_{\omega, s,+}^{(m)}$ be the space $\mathcal{L}_{\omega, s,+}^{(m)}$, where in the linear hull we omit the singular function defined by $\alpha \in]-1,-1 / 2[$. An easy computation shows that if $(f, g) \in$ $H_{0}^{s}\left(\mathbb{R}_{+}\right)^{2}+\chi \widetilde{\mathcal{L}}_{\omega, s,+}^{(m)}$, then

$$
S_{\omega}(z)\left(\begin{array}{c}
M f(z) \\
M g(z)
\end{array}\right)
$$

satisfies the hypothesis of Theorem 4 in [14] and hence is the Mellin transform of an element of $H^{s}\left(\mathbb{R}_{+}\right)^{2}$. In the same way, if $s-\frac{1}{2} \notin e_{\omega}^{(m)}$ and $(f, g) \in H^{s}\left(\mathbb{R}_{+}\right)^{2}$, we obtain that

$$
S_{\omega}(z)^{-1}\left(\begin{array}{c}
M f(z) \\
M g(z)
\end{array}\right)
$$

is the Mellin transform of an element of $H_{0}^{s}\left(\mathbb{R}_{+}\right)^{2}+\chi \mathcal{L}_{\omega, s,+}^{(m)}$.

Let us prove the inequality. By density, we can assume that $v=(f, g) \in$ $C_{0}^{\infty}\left(\mathbb{R}_{+}\right)^{2}$. We have

$$
\begin{aligned}
\left\langle D^{m} \mathcal{W}_{\omega} v,\right. & \left.D^{m} v\right\rangle_{L^{2}\left(\mathbb{R}_{+}\right)^{2}} \\
= & \left\|D^{m} f\right\|_{L^{2}\left(\mathbb{R}_{+}\right)^{2}}^{2}+\left\|D^{m} g\right\|_{L^{2}\left(\mathbb{R}_{+}\right)^{2}}^{2} \\
& +\left\langle D^{m} W g, D^{m} f\right\rangle_{L^{2}\left(\mathbb{R}_{+}\right)}+\left\langle D^{m} W f, D^{m} g\right\rangle_{L^{2}\left(\mathbb{R}_{+}\right)} .
\end{aligned}
$$

Moreover

$$
\begin{aligned}
M\left(D^{m}\right. & W f)(z) \\
& =(1-z)(2-z) \cdots(m-z) M(W f)(z-m) \\
& =(1-z)(2-z) \cdots(m-z) \frac{\cos ((z-m-1)(\pi-\omega))}{\sin (\pi(z-m))} M f(z-m) \\
& =(-1)^{m} \frac{\cos ((z-m-1)(\pi-\omega))}{\sin (\pi z)} M\left(D^{m} f\right)(z) .
\end{aligned}
$$

Hence

$$
\left\|D^{m} W f\right\|_{L^{2}\left(\mathbb{R}_{+}\right)}^{2}=\frac{1}{2 \pi} \int_{\mathbb{R}}\left|\frac{\cos \left(\left(\frac{1}{2}+m-i x\right)(\pi-\omega)\right)}{\sin \left(\pi\left(\frac{1}{2}+i x\right)\right)}\right|^{2}\left|M\left(D^{m} f\right)\left(\frac{1}{2}+i x\right)\right|^{2} d x .
$$


Let us prove that

$$
\left|\frac{\cos \left(\left(\frac{1}{2}+m-i x\right)(\pi-\omega)\right)}{\sin \left(\pi\left(\frac{1}{2}+i x\right)\right)}\right| \leq \delta=\sup \left(\left|1-\frac{\omega}{\pi}\right|,\left|\sin \left(\left(m+\frac{1}{2}\right) \omega\right)\right|\right) .
$$

This is obvious if $x=0$ or if $x$ is large enough, since the left hand side converges to 0 at infinity. We have

$$
\left|\frac{\cos \left(\left(\frac{1}{2}+m-i x\right)(\pi-\omega)\right)}{\sin \left(\pi\left(\frac{1}{2}+i x\right)\right)}\right|^{2}=\frac{\cosh (2 x(\pi-\omega))-\cos ((2 m+1) \omega)}{1+\cosh (2 \pi x)} .
$$

At a point where the derivative of this function vanishes, the value is

$$
\left(1-\frac{\omega}{\pi}\right) \frac{\sinh (2(\pi-\omega) x)}{\sinh (2 \pi x)} \leq\left(1-\frac{\omega}{\pi}\right)^{2},
$$

since $\sinh (a) / \sinh (b) \leq a / b$ if $0 \leq a<b$.

If $m-\frac{1}{2} \notin e_{\omega}^{(m)}$, the bound $\delta$ is strictly smaller than 1 . We get

$$
\left\langle D^{m} \mathcal{W}_{\omega} v, D^{m} v\right\rangle_{L^{2}\left(\mathbb{R}_{+}\right)^{2}} \geq(1-\delta)\left(\left\|D^{m} f\right\|^{2}+\left\|D^{m} g\right\|^{2}\right) .
$$

This proves the proposition.

5.2. Dirichlet and Neumann corners. We proceed in the same way in the case of a pure Dirichlet or Neumann corner. The results and the proofs are similar to the ones obtained above, but we give the precise results for the sake of completeness and later use.

We use the same notations as in the previous section, and first consider a pure Dirichlet corner. In the boundary values of (3), the contribution of the integral on one side to the value on the other side is

$$
\frac{x \sin \omega}{\pi} \int_{0}^{+\infty} \frac{h(y)}{x^{2}+y^{2}-2 x y \cos \omega} d y \text {. }
$$

Since

$$
D_{x}\left(\frac{x \sin \omega}{x^{2}+y^{2}-2 x y \cos \omega}\right)=-D_{y}\left(\frac{y \sin \omega}{x^{2}+y^{2}-2 x y \cos \omega}\right),
$$

we get

$$
D_{x}\left(\frac{x \sin \omega}{\pi} \int_{0}^{+\infty} \frac{h(y)}{x^{2}+y^{2}-2 x y \cos \omega} d y\right)=\frac{\sin \omega}{\pi} \int_{0}^{+\infty} \frac{y D h(y)}{x^{2}+y^{2}-2 x y \cos \omega} d y
$$

for any $h \in \bar{C}_{0}^{\infty}\left(\mathbb{R}_{+}\right)$, and hence by density for any $h \in \widetilde{H}^{1 / 2}\left(\mathbb{R}_{+}\right)$.

Since $(t, \nu)$ is a positive basis, we have $\partial_{t}=-D_{x}$ on $\Gamma_{2}$ and $\partial_{t}=D_{x}$ on $\Gamma_{1}$. Hence, the previous computations show that the equality

$$
\mathcal{D}_{\omega}\left(\begin{array}{l}
f_{1} \\
f_{2}
\end{array}\right):=T_{+}\left(f_{1}, f_{2}\right)=\left(\begin{array}{c}
\partial_{t} u_{\mid \Gamma_{1}} \\
\partial_{t} u_{\mid \Gamma_{2}}
\end{array}\right)=\frac{1}{2}\left(\begin{array}{cc}
I & -N \\
-N & I
\end{array}\right)\left(\begin{array}{l}
f_{1} \\
f_{2}
\end{array}\right)
$$

holds with $\Gamma_{1}=\mathbb{R}, \Gamma_{2}=e^{i \omega} \mathbb{R}, u=K(g, h), \partial_{t} h=f, f_{j}=f_{\mid \Gamma_{j}}$ and

$$
N f(x)=\frac{\sin \omega}{\pi} \int_{0}^{+\infty} \frac{y f(y)}{x^{2}+y^{2}-2 x y \cos \omega} d y
$$

We get exactly the same operator in a pure Neumann corner. For $T_{-}$, we just have to change the sign of the identity. 
Proposition 8. If $\omega \in] 0,2 \pi\left[\backslash\{\pi\}, s \geq 0\right.$ and $\chi \in C_{0}^{\infty}(\mathbb{R})$ is equal to 1 in a neighborhood of 0 , then the operator

$$
\mathcal{D}_{\omega}: H_{0}^{s}\left(\mathbb{R}_{+}\right)^{2}+\chi \mathcal{L}_{\omega, s,+}^{(p)} \rightarrow H^{s}\left(\mathbb{R}_{+}\right)^{2}
$$

is continuous. Moreover, if $s-\frac{1}{2} \notin e_{\omega}^{(p)}$, it is bijective. If $m$ is an integer and $m-\frac{1}{2} \notin e_{\omega}^{(p)}$, then there is a $c>0$ such that

$$
\left\langle D^{m} \mathcal{D}_{\omega} v, D^{m} v\right\rangle_{L^{2}\left(\mathbb{R}_{+}\right)^{2}} \geq c\left\|D^{m} v\right\|_{L^{2}\left(\mathbb{R}_{+}\right)^{2}}^{2}
$$

for any $v \in H_{0}^{m}\left(\mathbb{R}_{+}\right)^{2}$.

The proof is analogous to that of Theorem 7 For later references, let us compute the Mellin transform of $N f$. Using the same techniques as above, we obtain

$$
\begin{aligned}
M(N f)(z) & =\frac{\sin \omega}{\pi} \int_{0}^{+\infty} x^{z-1} d x \int_{0}^{+\infty} \frac{y f(y)}{x^{2}+y^{2}-2 x y \cos \omega} d y \\
& =\frac{\sin \omega}{\pi} \int_{0}^{+\infty} y f(y) d y \int_{0}^{+\infty} \frac{x^{z-1}}{x^{2}+y^{2}-2 x y \cos \omega} d x
\end{aligned}
$$

Hence

$$
M(N f)(z)=\frac{\sin ((z-1)(\pi-\omega))}{\sin (\pi(z-1))} M f(z)
$$

if $\Re z=\frac{1}{2}$.

We also need a commutation property with the multiplication by a smooth function.

Proposition 9. If $\omega \in] 0,2 \pi\left[\backslash\{\pi\}, m \in \mathbb{N}\right.$ and $\chi \in C_{0}^{\infty}(\mathbb{R})$, then the operators $\chi \mathcal{W}_{\omega}-\mathcal{W}_{\omega} \chi$ and $\chi \mathcal{D}_{\omega}-\mathcal{D}_{\omega} \chi$ map $H_{0}^{m}\left(\mathbb{R}_{+}\right)^{2}$ continuously into $H^{m+1}\left(\mathbb{R}_{+}\right)^{2}$.

Proof. We treat the case of $\mathcal{W}_{\omega}$. The other one is similar. Since $W$ is continuous from $H_{0}^{m}\left(\mathbb{R}_{+}\right)$to $H^{m}\left(\mathbb{R}_{+}\right)$, we have to prove that there is $C>0$ such that

$$
\left\|D\left(\chi D^{m} W f-D^{m} W(\chi f)\right)\right\|_{L^{2}\left(\mathbb{R}_{+}\right)} \leq C\|f\|_{H^{m}\left(\mathbb{R}_{+}\right)}
$$

for every $f \in C_{0}^{\infty}\left(\mathbb{R}_{+}\right)$. By definition of $W$, we get

$$
\begin{aligned}
D^{m}(W f)(x) & =\frac{1}{\pi} D_{x}^{m} \int_{0}^{+\infty} \Re\left(\frac{e^{i \omega}}{x e^{i \omega}-y}\right) f(y) d y \\
& =\frac{1}{\pi} \int_{0}^{+\infty} \Re\left(\left(-D_{y}\right)^{m} \frac{e^{i(m+1) \omega}}{x e^{i \omega}-y}\right) f(y) d y \\
& =\frac{1}{\pi} \int_{0}^{+\infty} \Re\left(\frac{e^{i(m+1) \omega}}{x e^{i \omega}-y}\right) D^{m} f(y) d y .
\end{aligned}
$$

Hence, we have to estimate the derivative of

$$
\int_{0}^{+\infty} \Re\left(\frac{e^{i(m+1) \omega}}{x e^{i \omega}-y}\right)\left[(\chi(x)-\chi(y)) D^{m} f(y)-\left(D^{m}(\chi f)(y)-\chi(y) D^{m} f(y)\right)\right] d y .
$$

The last two terms involve derivatives of $f$ of order strictly less than $m$. We can make a further integration by parts and conclude by a classical lemma concerning 
the continuity of operator with -1-homogeneous kernel ([11], p. 229). For the first two terms, we notice that, since the function $\chi$ is a Lipschitz function,

$$
\begin{aligned}
& \left|\int_{0}^{+\infty}(\chi(x)-\chi(y)) D_{x} \Re\left(\frac{e^{i(m+1) \omega}}{x e^{i \omega}-y}\right) D^{m} f(y) d y\right| \\
& \quad \leq C \int_{0}^{+\infty}\left|\Re\left(\frac{e^{i(m+2) \omega}}{\left(x e^{i \omega}-y\right)^{2}}\right)\right||x-y|\left|D^{m} f(y)\right| d y
\end{aligned}
$$

and we use the same lemma.

\section{The Fredholm PRoperty of the Boundary operator}

In the remaining sections, we fix a bounded polygonal open subset $\Omega$ of $\mathbb{R}^{2}$ with a connected boundary $\Gamma$ and use the notations of Section 2

If $x, y \in \Gamma$ are not corners and $x \neq y$, then

$$
\partial_{\nu_{x}} \frac{(x-y) \cdot \nu_{y}}{|x-y|^{2}}=\partial_{t_{y}} \frac{(x-y) \cdot t_{x}}{|x-y|^{2}} .
$$

This follows easily from the fact that if $\left(u_{1}, u_{2}\right)$ and $\left(v_{1}, v_{2}\right)$ are two positive bases of $\mathbb{R}^{2}$, then

$$
\left(u_{1} \cdot v_{1}+u_{2} \cdot v_{2}\right)|x|^{2}=2\left(x \cdot u_{1} x \cdot v_{1}+x \cdot u_{2} x \cdot v_{2}\right) .
$$

It follows that the operator $T_{ \pm}$maps $(f, g)$ to

$$
\pm \frac{f(x)}{2}+\frac{1}{2 \pi} \int_{\Gamma_{D}} \frac{(x-y) \cdot \nu_{x}}{|x-y|^{2}} f(y) d \sigma(y)+\frac{1}{2 \pi} \int_{\Gamma_{N}} \frac{(x-y) \cdot t_{x}}{|x-y|^{2}} g(y) d \sigma(y)
$$

on $\Gamma_{D}$, and to

$$
\pm \frac{g(x)}{2}+\frac{1}{2 \pi} \int_{\Gamma_{N}} \frac{(x-y) \cdot \nu_{x}}{|x-y|^{2}} g(y) d \sigma(y)-\frac{1}{2 \pi} \int_{\Gamma_{D}} \frac{(x-y) \cdot t_{x}}{|x-y|^{2}} f(y) d \sigma(y)
$$

on $\Gamma_{N}$. In some sense, we have gotten a symmetry for the contributions of $f$ and $g$.

As in Section 2 let us denote by $L_{v}^{2}\left(\Gamma_{D}\right)$ the subset of $L^{2}\left(\Gamma_{D}\right)$ formed by the functions whose integrals on each connected component of $\Gamma_{D}$ vanish.

Proof of Theorem [1. It follows from Propositions 7 and 8 that $T_{ \pm}$maps $\mathcal{H}_{ \pm}^{s}\left(\Gamma_{D}, \Gamma_{N}\right)$ into $H_{c}^{s}\left(\Gamma_{D}\right) \times H_{c}^{s}\left(\Gamma_{N}\right)$ if $s \geq 0$.

Choose functions $\psi_{j}$ on the boundary $\Gamma$ which are restrictions of functions of $C_{0}^{\infty}\left(\mathbb{R}^{2}\right)$ and such that $\psi_{j}$ is equal to $\delta_{j k}$ near $P_{k}$ for every $k$. We may assume that

$$
\operatorname{supp}\left(\psi_{j}\right) \subset \Gamma_{j} \cup \Gamma_{j-1}, \quad \sum_{j=0}^{M-1} \psi_{j}^{2}=1 .
$$

Let $(f, g) \in L_{v}^{2}\left(\Gamma_{D}\right) \times L^{2}\left(\Gamma_{N}\right)$. We can identify the pair $(f, g)$ to a unique function $F \in L^{2}(\Gamma)$. Using Proposition 9 we write

$$
\begin{aligned}
\left\langle T_{ \pm}(f, g),(f, g)\right\rangle_{L^{2}\left(\Gamma_{D}\right) \times L^{2}\left(\Gamma_{N}\right)}=\left\langle T_{ \pm} F, F\right\rangle_{L^{2}(\Gamma)} & \\
= & \sum_{j, k=0}^{M-1}\left\langle T_{ \pm}\left(\psi_{j}^{2} F\right), \psi_{k}^{2} F\right\rangle_{L^{2}(\Gamma)} \\
= & \sum_{j, k=0}^{M-1}\left\langle T_{ \pm}\left(\psi_{j} \psi_{k} F\right), \psi_{j} \psi_{k} F\right\rangle_{L^{2}(\Gamma)}-\langle K F, F\rangle_{L^{2}(\Gamma)},
\end{aligned}
$$


where $K$ is a continuous operator from $L^{2}(\Gamma)$ into $H^{1}(\Gamma)$. Since $\omega_{j} \neq \pi$ for any $j$, it follows that $-\frac{1}{2} \notin \bigcup_{j} e_{\omega_{j}}$. Denote by $\mathcal{W}_{j}$ the matrix of operators of Propositions 7 or 8 associated to the $j$-th corner according to the nature of this corner, see Section 5. In the interior (resp. exterior) case the diagonal blocks are $I$ (resp. $-I$ ). Using the second part of Propositions 7 and 8 , we get

$$
\begin{aligned}
& \pm\left\langle T_{ \pm}(f, g),(f, g)\right\rangle_{L^{2}\left(\Gamma_{D}\right) \times L^{2}\left(\Gamma_{N}\right)} \\
& \quad \sum_{j, k=0}^{M-1}\left\langle \pm \mathcal{W}_{j}\left(\psi_{j} \psi_{k} F\right), \psi_{j} \psi_{k} F\right\rangle_{L^{2}(\Gamma)}-\langle K F, F\rangle_{L^{2}(\Gamma)} \\
& \geq c \sum_{j, k=0}^{M-1}\left\|\psi_{j} \psi_{k} F\right\|_{L^{2}(\Gamma)}^{2}-\langle K F, F\rangle_{L^{2}(\Gamma)} \\
& \geq c\left(\|f\|_{L^{2}\left(\Gamma_{D}\right)}^{2}+\|g\|_{L^{2}\left(\Gamma_{N}\right)}^{2}\right)-\langle K F, F\rangle_{L^{2}(\Gamma)}
\end{aligned}
$$

for some $c>0$.

The operator $K$ is compact from $L^{2}(\Gamma)$ into itself. Moreover, $L_{v}^{2}\left(\Gamma_{D}\right) \times L^{2}\left(\Gamma_{N}\right)$ has codimension $2 p$ in $\mathcal{H}_{ \pm}^{0}\left(\Gamma_{D}, \Gamma_{N}\right)$ and also in $L^{2}\left(\Gamma_{D}\right) \times L^{2}\left(\Gamma_{N}\right) \times \mathbb{C}^{p}$. Using Lemma 10, it follows that

$$
\mathcal{T}_{+}: \mathcal{H}_{+}^{0}\left(\Gamma_{D}, \Gamma_{N}\right) \rightarrow L^{2}\left(\Gamma_{D}\right) \times L^{2}\left(\Gamma_{N}\right) \times \mathbb{C}^{p}:(f, g) \mapsto\left(T_{+}(f, g), S_{+}(f, g)\right)
$$

and

$$
\begin{aligned}
\mathcal{T}_{-}: \mathcal{H}_{-}^{0}\left(\Gamma_{D}, \Gamma_{N}\right) \times \mathbb{C} & \rightarrow L^{2}\left(\Gamma_{D}\right) \times L^{2}\left(\Gamma_{N}\right) \times \mathbb{C}^{p+1} \\
(f, g, c) & \mapsto\left(T_{-}(f, g), S_{-}(f, g), \int_{\Gamma_{N}} g d \sigma\right),
\end{aligned}
$$

are Fredholm operators with index 0 .

If $\Gamma_{D}=\emptyset$, it is well known, see 18, that the kernel of $\mathcal{T}_{+}$is one dimensional. Assume that $\Gamma_{D} \neq \emptyset$ and that $(f, g)$ is in the kernel of this operator. It follows that the function (3) defined by $(f, g)$ solves the mixed problem with null data. Hence it vanishes in $\Omega$. By the last part of Proposition [6, we get

$$
g=-\frac{\partial_{\nu} G}{2 \pi} \int_{\Gamma_{N}} g d \sigma, \quad h=-\frac{G}{2 \pi} \int_{\Gamma_{N}} g d \sigma .
$$

By the first part of the same proposition, it follows that

$$
\log \gamma \int_{\Gamma_{N}} g d \sigma=0
$$

This shows that $\mathcal{T}_{+}$is one to one if $\gamma \neq 1$, and that the kernel is one dimensional if $\gamma=1$.

Let us consider the case of $\mathcal{T}_{-}$. If $\Gamma_{D}=\emptyset$, it follows from the results of 18 that the kernel of $\mathcal{T}_{-}$is $\{(0, c): c \in \mathbb{C}\}$. Assume that $\Gamma_{D} \neq \emptyset$ and choose $(f, g, c)$ in the kernel of $\mathcal{T}_{-}$. It follows that the function $u$ defined by (4) vanishes in $\mathbb{R}^{2} \backslash \bar{\Omega}$, since it satisfies the mixed boundary conditions and is bounded at infinity. Let us consider the same function inside $\Omega$. On $\Gamma_{D}$, its normal derivative vanishes, since it vanishes outside $\bar{\Omega}$ and the normal derivative of the double layer potential is continuous across $\Gamma_{D}$; see Lemma 4.1 of [2]. On $\Gamma_{N}$, it vanishes since it vanishes outside $\bar{\Omega}$ and the single layer potential is continuous across $\Gamma_{N}$. Since $\Gamma_{N} \neq \emptyset$, the mixed problem in $\Omega$ has a unique solution; hence (44) also vanishes in $\Omega$. Using Proposition [6 and the fact that the integral of $g$ is zero, we obtain $f=g=c=0$. 
It follows from the local results of Propositions 7 and 8 that the inverse of $\mathcal{T}_{+}$ (resp. $\left.\mathcal{T}_{-}\right)$maps $H_{c}^{s}\left(\Gamma_{D}\right) \times H_{c}^{s}\left(\Gamma_{N}\right) \times \mathbb{C}^{p}\left(\right.$ resp. $\left.H_{c}^{s}\left(\Gamma_{D}\right) \times H_{c}^{s}\left(\Gamma_{N}\right) \times \mathbb{C}^{p+1}\right)$ into $\mathcal{H}_{+}^{s}\left(\Gamma_{D}, \Gamma_{N}\right)\left(\right.$ resp. $\left.\mathcal{H}_{-}^{s}\left(\Gamma_{D}, \Gamma_{N}\right) \times \mathbb{C}\right)$. This proves the theorem.

We have used the following elementary lemma in Fredholm theory.

Lemma 10. Let $E, F$ be two Hilbert spaces, $L$ a Hilbert space which is a closed subspace of $E$ and $F$ with finite codimensions, and $T \in L(E ; F)$. If there is $c>0$ such that

$$
\langle T f, f\rangle \geq c\|f\|^{2}
$$

for any $f \in L$, then $T$ is a Fredholm operator and

$$
\operatorname{ind}(T)=\operatorname{codim}_{E} L-\operatorname{codim}_{F} L .
$$

\section{Convergence of the methods}

Proof of Theorem Q We give the proof for the interior problem. The exterior case is similar. We apply Lemma 11 to the operator

$$
\mathcal{T}_{+}: \mathcal{H}_{+}^{m}\left(\Gamma_{D}, \Gamma_{N}\right) \rightarrow H_{c}^{m}\left(\Gamma_{D}\right) \times H_{c}^{m}\left(\Gamma_{N}\right) \times \mathbb{C}^{p}:(f, g) \mapsto\left(T_{+}(f, g), S_{+}(f, g)\right) .
$$

By Theorem 1, this is an isomorphism. We use the spaces $V_{\Delta, 0, \pm}^{(m)}(\Gamma)$ as trial spaces.

The test spaces are defined in the following way. On

$$
Y_{m}=H_{c}^{m}\left(\Gamma_{D}\right) \times H_{c}^{m}\left(\Gamma_{N}\right) \times \mathbb{C}^{p},
$$

we consider the norm

$$
\begin{aligned}
\|(u, v, c)\|_{Y_{m}}^{2}= & |c|^{2}+\sum_{j \in e_{D}}\left(\sum_{k=0}^{m-1}\left|\partial_{+}^{k} u\left(P_{j}\right)\right|^{2}+\left\|\partial_{t}^{m} u\right\|_{L^{2}\left(\Gamma_{j}\right)}^{2}\right) \\
& +\sum_{j \in e_{N}}\left(\sum_{k=0}^{m-1}\left|\partial_{+}^{k} v\left(P_{j}\right)\right|^{2}+\left\|\partial_{t}^{m} v\right\|_{L^{2}\left(\Gamma_{j}\right)}^{2}\right) .
\end{aligned}
$$

Denote by $S_{\Delta}^{(m)}(\Gamma)$ the space of functions which are smoothest splines of degree $2 m-1$ subordinated to the mesh $\Delta$ on each side $\Gamma_{j}$. As test space, we use the linear hull $U_{\Delta}^{(m)}(\Gamma)$ of the linear forms

$$
H_{c}^{m}\left(\Gamma_{D}\right) \times H_{c}^{m}\left(\Gamma_{N}\right) \times \mathbb{C}^{p} \rightarrow \mathbb{C}:(u, v, c) \mapsto\left\langle(u, v, c),\left(S_{1}, S_{2}, \alpha\right)\right\rangle_{Y_{m}}
$$

with

$$
\left(S_{1}, S_{2}, \alpha\right) \in S_{\Delta}^{(m)}(\Gamma) \times S_{\Delta}^{(m)}(\Gamma) \times \mathbb{C}^{p} .
$$

The dimension of this space is

$$
\operatorname{dim} U_{\Delta}^{(m)}(\Gamma)=p+\sum_{j=0}^{M-1}\left(n_{j}+1+2(m-1)\right)=\operatorname{dim} V_{\Delta, 0, \pm}^{(m)}(\Gamma) .
$$

The collocation equations (6) are equivalent to

$$
\omega\left(\mathcal{T}_{+}\left(\partial_{t} h_{\Delta}, g_{\Delta}\right)\right)=\omega\left(\mathcal{T}_{+}\left(\partial_{t} h, g\right)\right), \quad \omega \in U_{\Delta}^{(m)}(\Gamma) .
$$

Indeed, a linear form $\omega$ involves a scalar product in $Y_{m}$ with a function which is a smoothest spline on each side. Let us perform $m$ integrations by parts in the $L^{2}$ parts of the scalar product to obtain derivatives of order $2 m$ of the splines. We get sums of multiples of the Dirac masses at the collocation points and also 
of derivatives $\partial_{ \pm, j}^{\ell}$ of order strictly less than $m$ at the points $P_{j}$. The equations involving the points $Q_{j}$ are generated by the $|c|$ part of the $Y_{m}$ norm. This shows that the equations (15) are consequences of the equations (6). Since the dimension of $U_{\Delta}^{(m)}(\Gamma)$ is the number of equations in (6), we have equivalence.

Let $\Delta_{k}$ be a sequence of meshes whose meshwidths converge to 0 . It follows from the approximation properties of splines in Sobolev spaces, see for example [16, that the orthogonal projections onto $U_{\Delta_{k}}^{(m)}(\Gamma)$ converge strongly to the identity of $Y_{m}^{\prime}$. It remains to prove that there are $\delta>0$ and a compact operator $K$ on $\mathcal{H}_{+}^{m}\left(\Gamma_{D}, \Gamma_{N}\right)$ such that

$$
\sup _{v \in U_{\Delta_{k}}^{(m)}(\Gamma),\|v\|_{Y_{m}^{\prime}}=1}\left|\left\langle v, \mathcal{T}_{+} u\right\rangle\right| \geq \delta\|u\|_{\mathcal{H}_{+}^{m}\left(\Gamma_{D}, \Gamma_{N}\right)}-\|K u\|_{\mathcal{H}_{+}^{m}\left(\Gamma_{D}, \Gamma_{N}\right)}
$$

for any $k$ and $u \in V_{\Delta_{k}, 0,+}^{(m)}(\Gamma)$.

The argument is similar to the one used in the first part of the proof of Theorem 1 Choose functions $\psi_{j}$ on the boundary $\Gamma$ as in that proof. Let $u=(f, g) \in$ $V_{\Delta_{k}, 0,+}^{(m)}(\Gamma)$. Write

$$
u=S+F \text { with } S=\left(S_{D}, S_{N}\right) \in S_{\Delta, 0}^{(m)}(\Gamma) \text { and } F \in \sum_{j=0}^{M-1} \chi_{j} \mathcal{L}_{j, 2 m-1}^{+}
$$

and consider the form

$$
\omega: H_{c}^{m}\left(\Gamma_{D}\right) \times H_{c}^{m}\left(\Gamma_{N}\right) \times \mathbb{C}^{p} \rightarrow \mathbb{C}:\left(f_{D}, f_{N}, c\right) \mapsto\left\langle\left(f_{D}, f_{N}, c\right),\left(S_{D}, S_{N}, 0\right)\right\rangle_{Y_{m}} .
$$

Since the function $S$ vanishes at the corners to order $2 m-1$, it follows that

$$
\begin{aligned}
\left\langle\omega, \mathcal{T}_{+} u\right\rangle & \\
= & \left\langle\partial_{t}^{m} T_{+} S, \partial_{t}^{m} S\right\rangle_{L^{2}(\Gamma)}+\left\langle\partial_{t}^{m} T_{+} F, \partial_{t}^{m} S\right\rangle_{L^{2}(\Gamma)} \\
& =\sum_{j, k=0}^{M-1}\left\langle\partial_{t}^{m} T_{+}\left(\psi_{j}^{2} S\right), \partial_{t}^{m}\left(\psi_{k}^{2} S\right)\right\rangle_{L^{2}(\Gamma)}+\left\langle\partial_{t}^{m} T_{+} F, \partial_{t}^{m} S\right\rangle_{L^{2}(\Gamma)}
\end{aligned}
$$

By Proposition 9 the commutators $T_{+} \psi_{j}-\psi_{j} T_{+}$are continuous operators from $H_{0}^{m}(\Gamma)$ into $H_{c}^{m+1}(\Gamma)$. Using the explicit form (13) and (14) of $T_{+}$, Propositions 7 and 8 and the fact that $\sum_{j=0}^{M-1} \chi_{j} \mathcal{L}_{j, 2 m-1}^{+}$is finite dimensional, we obtain

$$
\begin{aligned}
\left\langle\omega, \mathcal{T}_{+} u\right\rangle & \\
= & \sum_{j, k=0}^{M-1}\left\langle\partial_{t}^{m} T_{+}\left(\psi_{j} \psi_{k} S\right), \partial_{t}^{m}\left(\psi_{j} \psi_{k} S\right\rangle_{L^{2}(\Gamma)}\right. \\
& \quad+\left\langle\partial_{t}^{m} T_{+} F, \partial_{t}^{m} S\right\rangle_{L^{2}(\Gamma)}-\left\|K_{1} S\right\|_{\mathcal{H}_{+}^{m}\left(\Gamma_{D}, \Gamma_{N}\right)}\left\|\partial_{t}^{m} S\right\|_{L^{2}(\Gamma)} \\
& \\
\geq & \delta \sum_{j, k=1}^{M-1}\left\|\partial_{t}^{m}\left(\psi_{j} \psi_{k} S\right)\right\|_{L^{2}(\Gamma)}^{2}-\left\|K_{2} u\right\|_{\mathcal{H}_{+}^{m}\left(\Gamma_{D}, \Gamma_{N}\right)}\left\|\partial_{t}^{m} S\right\|_{L^{2}(\Gamma)}
\end{aligned}
$$


where $K_{1}, K_{2}$ are compact operators on $\mathcal{H}_{+}^{m}\left(\Gamma_{D}, \Gamma_{N}\right)$ and $\delta>0$. We have

$$
\begin{aligned}
\left\|\partial_{t}^{m} S\right\|_{L^{2}(\Gamma)}^{2} & =\left\|\sum_{j=0}^{M-1} \partial_{t}^{m}\left(\psi_{j}^{2} S\right)\right\|_{L^{2}(\Gamma)}^{2} \\
& \leq M \sum_{j=0}^{M-1}\left\|\partial_{t}^{m}\left(\psi_{j}^{2} S\right)\right\|_{L^{2}(\Gamma)}^{2}
\end{aligned}
$$

and

$$
\|\omega\|_{Y_{m}^{\prime}} \leq\left\|\partial_{t}^{m} S\right\|_{L^{2}(\Gamma)}
$$

It follows that

$$
\begin{aligned}
\sup _{v \in U_{\Delta_{k}}^{(m)}(\Gamma),\|v\|_{Y_{m}^{\prime}}=1}\left|\left\langle v, \mathcal{T}_{+} u\right\rangle\right| & \geq \frac{\delta}{M}\left\|\partial_{t}^{m} S\right\|_{L^{2}(\Gamma)}-\left\|K_{2} u\right\|_{\mathcal{H}_{+}^{m}\left(\Gamma_{D}, \Gamma_{N}\right)} \\
& \geq \delta^{\prime}\|u\|_{\mathcal{H}_{+}^{m}\left(\Gamma_{D}, \Gamma_{N}\right)}-\left\|K_{3} u\right\|_{\mathcal{H}_{+}^{m}\left(\Gamma_{D}, \Gamma_{N}\right)},
\end{aligned}
$$

since $H_{0}^{m}\left(\Gamma_{D}\right) \times H_{0}^{m}\left(\Gamma_{D}\right)$ has finite codimension in $\mathcal{H}_{+}^{m}\left(\Gamma_{D}, \Gamma_{N}\right)$ and the norm in $H_{0}^{m}\left(\Gamma_{j}\right)$ is equivalent to $\left\|\partial_{t}^{m} f\right\|_{L^{2}\left(\Gamma_{j}\right)}$. This proves the theorem.

We have used the following formulation of Céa's lemma. For the sake of completeness we give a short proof, see also [4] or [16].

Lemma 11 (Céa). Let $X$ and $Y$ be Banach spaces, let $A \in L(X ; Y)$ be continuous and bijective, and let $V_{n} \subset X, T_{n} \subset Y^{\prime}$ be sequences of subspaces satisfying $\operatorname{dim} V_{n}=\operatorname{dim} T_{n}<+\infty$. Assume that

(i) there are linear continuous operators $P_{n}$ from $Y^{\prime}$ on $T_{n}$ satisfying $P_{n} f \rightarrow f$ in $Y^{\prime}$ for every $f \in Y^{\prime}$, and

(ii) there are $\delta>0$ and a compact operator $K \in L(X ; X)$ such that

$$
\sup _{v \in T_{n},\|v\|_{Y^{\prime}}=1}|\langle v, A u\rangle| \geq \delta\|u\|_{X}-\|K u\|_{X}
$$

for every $u \in V_{n}$.

Then, there is $n_{0}>0$ such that, if $n \geq n_{0}$, the equation

$$
\left\langle v, A u_{n}\right\rangle=\langle v, A u\rangle, \quad v \in T_{n},
$$

has a unique solution $u_{n} \in V_{n}$ for every $u \in X$. Moreover, there is $C>0$ such that

$$
\left\|u-u_{n}\right\|_{X} \leq C \inf _{w \in V_{n}}\|u-w\|_{X} .
$$

Proof. If $K=0$, condition (ii) shows that the equation defining $u_{n}$ has a unique solution. Moreover

$$
\begin{aligned}
\delta\left\|u_{n}\right\|_{X} & \leq \sup _{v \in T_{n},\|v\|_{Y^{\prime}}=1}\left|\left\langle v, A u_{n}\right\rangle\right| \\
& =\sup _{v \in T_{n},\|v\|_{Y^{\prime}}=1}|\langle v, A u\rangle| \leq\|A\|\|u\|_{X} .
\end{aligned}
$$

Hence, for every $w \in V_{n}$, we have

$$
\begin{aligned}
\left\|u-u_{n}\right\|_{X} & =\left\|u-w-(u-w)_{n}\right\|_{X} \\
& \leq\left(1+\frac{\|A\|}{\delta}\right)\|u-w\|_{X} .
\end{aligned}
$$

This proves the lemma if $K=0$. 
Let us show that, for large $n$, the estimation (ii) is valid with $K=0$ and a smaller constant $\delta$. Let $u \in V_{n}$. There is $v \in T_{n}$ such that $\|v\|_{Y^{\prime}}=1$ and

$$
\langle v, A u\rangle \geq \delta\|u\|_{X}-\|K u\|_{X} .
$$

By the Hahn-Banach theorem, there is $w \in X^{\prime}$ such that $\|w\|_{X^{\prime}} \leq 1$ and $\langle w, K u\rangle=$ $\|K u\|_{X}$. Write

$$
\left\langle v+P_{n} A^{*-1} K^{*} w, A u\right\rangle=\langle v, A u\rangle+\|K u\|_{X}-\left\langle\left(I-P_{n}\right) A^{*-1} K^{*} w, A u\right\rangle .
$$

Since $I-P_{n}$ converges strongly to 0 and $K^{*}$ is compact, it follows that the operators $A^{*}\left(I-P_{n}\right) A^{*-1} K^{*}$ converge to 0 in operator norm. If $\delta_{n}$ is the norm of this operator, we get

$$
\left|\left\langle v+P_{n} A^{*-1} K^{*} w, A u\right\rangle\right| \geq\left(\delta-\delta_{n}\right)\|u\|_{X} .
$$

Moreover, since the operators $P_{n}$ are uniformly bounded, we have

$$
\left\|v+P_{n} A^{*-1} K^{*} w\right\|_{Y^{\prime}} \leq 1+C\left\|A^{-1}\right\|\|K\| .
$$

This proves the lemma.

For the proof of Theorem 3 we use an adaptation of the Aubin-Nitsche type estimates.

Proof of Theorem 3. We keep the same notations as in the proof of Theorem 2 and consider the interior case.

Let $\xi \in \mathcal{H}_{+}^{k}\left(\Gamma_{D}, \Gamma_{N}\right)^{\prime}$. Since $m-\frac{1}{2}, k-\frac{1}{2} \notin e_{\omega_{j}}$ for every $j$, it follows that the operator $\mathcal{T}_{+}$is an isomorphism from $\mathcal{H}_{+}^{m}\left(\Gamma_{D}, \Gamma_{N}\right)$ onto

$$
Y_{m}=H_{c}^{m}\left(\Gamma_{D}\right) \times H_{c}^{m}\left(\Gamma_{N}\right) \times \mathbb{C}^{p}
$$

and $\mathcal{H}_{+}^{m}\left(\Gamma_{D}, \Gamma_{N}\right) \subset \mathcal{H}_{+}^{k}\left(\Gamma_{D}, \Gamma_{N}\right)$. Hence $\mathcal{T}_{+}^{\prime-1}\left(\xi_{\mid \mathcal{H}_{+}^{m}\left(\Gamma_{D}, \Gamma_{N}\right)}\right) \in Y_{m}^{\prime}$. Using (15), write

$$
\begin{aligned}
\left\langle\xi,\left(\partial_{t} h, g\right)-\left(\partial_{t} h_{\Delta}, g_{\Delta}\right)\right\rangle & \\
= & \left\langle\mathcal{T}_{+}^{\prime-1}\left(\xi_{\mid \mathcal{H}_{+}^{m}\left(\Gamma_{D}, \Gamma_{N}\right)}\right), \mathcal{T}_{+}\left(\left(\partial_{t} h, g\right)-\left(\partial_{t} h_{\Delta}, g_{\Delta}\right)\right)\right\rangle \\
& =\left\langle\mathcal{T}_{+}^{\prime-1}\left(\xi_{\mid \mathcal{H}_{+}^{m}\left(\Gamma_{D}, \Gamma_{N}\right)}\right)-\omega, \mathcal{T}_{+}\left(\left(\partial_{t} h, g\right)-\left(\partial_{t} h_{\Delta}, g_{\Delta}\right)\right)\right\rangle
\end{aligned}
$$

for every $\omega \in U_{\Delta}^{(m)}(\Gamma)$. It follows from Theorem 2 that

$$
\begin{aligned}
\mid\left\langle\xi,\left(\partial_{t} h, g\right)-\right. & \left.\left(\partial_{t} h_{\Delta}, g_{\Delta}\right)\right\rangle \mid \\
\leq & \left\|\mathcal{T}_{+}\left(\left(\partial_{t} h, g\right)-\left(\partial_{t} h_{\Delta}, g_{\Delta}\right)\right)\right\|_{Y_{m}} \inf _{\omega \in U_{\Delta}^{(m)}(\Gamma)}\left\|\mathcal{T}_{+}^{\prime-1}\left(\xi_{\mid \mathcal{H}_{+}^{m}\left(\Gamma_{D}, \Gamma_{N}\right)}\right)-\omega\right\|_{Y_{m}^{\prime}} \\
\leq & C \inf _{(\tilde{g}, \tilde{h}) \in W_{\Delta,+}^{(m)}(\Gamma)}\left\|\left(\partial_{t} h, g\right)-\left(\partial_{t} \tilde{h}, \tilde{g}\right)\right\|_{\mathcal{H}_{+}^{m}\left(\Gamma_{D}, \Gamma_{N}\right)} \\
& \quad \times \inf _{\omega \in U_{\Delta}^{(m)}(\Gamma)}\left\|\mathcal{T}_{+}^{\prime-1}\left(\xi_{\mid \mathcal{H}_{+}^{m}\left(\Gamma_{D}, \Gamma_{N}\right)}\right)-\omega\right\|_{Y_{m}^{\prime}} .
\end{aligned}
$$

Let us transform the second factor. By the Riesz representation theorem, there is $\eta \in Y_{m}$ such that

$$
\left\langle\mathcal{T}_{+}^{\prime-1} \xi_{\mid \mathcal{H}_{+}^{m}\left(\Gamma_{D}, \Gamma_{N}\right)}, \varphi\right\rangle=\langle\eta, \varphi\rangle_{Y_{m}}, \quad \varphi \in Y_{m}
$$


Since the operator $\mathcal{T}_{+}$is also an isomorphism from $\mathcal{H}_{+}^{k}\left(\Gamma_{D}, \Gamma_{N}\right)$ onto $Y_{k}=H_{c}^{k}\left(\Gamma_{D}\right) \times$ $H_{c}^{k}\left(\Gamma_{N}\right) \times \mathbb{C}^{p}$, we get

$$
\begin{aligned}
\left|\langle\eta, \varphi\rangle_{Y_{m}}\right| & =\left|\left\langle\mathcal{T}_{+}^{\prime-1} \xi, \varphi\right\rangle\right|=\left|\left\langle\xi, \mathcal{T}_{+}^{-1} \varphi\right\rangle\right| \\
& \leq C\|\xi\|_{\mathcal{H}_{+}^{k}\left(\Gamma_{D}, \Gamma_{N}\right)^{\prime}}\|\varphi\|_{Y_{k}}
\end{aligned}
$$

for every $\varphi \in Y_{m}$. Now, for every $u \in Y_{k}$, there is one and only one $v \in Y_{m}$ such that

$$
\langle u, \varphi\rangle_{Y_{k}}=\langle v, \varphi\rangle_{Y_{m}}, \quad \varphi \in Y_{m}
$$

On each side $\Gamma_{j}$, we have $D^{2 m} v=D^{2 k} u$ in the distributional sense. Hence, $v \in$ $Y_{2 m-k}$. Moreover, by the closed graph theorem, the operator $Y_{k} \rightarrow Y_{2 m-k}: u \mapsto v$ is continuous. It follows that

$$
\eta \in Y_{2 m-k} \text { and }\|\eta\|_{Y_{2 m-k}} \leq C\|\xi\|_{\mathcal{H}_{+}^{k}\left(\Gamma_{D}, \Gamma_{N}\right)^{\prime}}
$$

where $C>0$ is independent of $\xi$.

The linear forms belonging to $U_{\Delta}^{(m)}(\Gamma)$ are defined as scalar products in $Y_{m}$ with spline functions. The norms are preserved. Hence, using the rate of convergence of smoothest splines in Sobolev spaces, see 14 or [16, we obtain

$$
\begin{aligned}
& \left|\left\langle\xi,\left(\partial_{t} h, g\right)-\left(\partial_{t} h_{\Delta}, g_{\Delta}\right)\right\rangle\right| \\
& \leq \quad \inf _{(\tilde{g}, \tilde{h}) \in W_{\Delta,+}^{(m)}(\Gamma)}\left\|\left(\partial_{t} h, g\right)-\left(\partial_{t} \tilde{h}, \tilde{g}\right)\right\|_{\mathcal{H}_{+}^{m}\left(\Gamma_{D}, \Gamma_{N}\right)} \\
& \quad \times \inf _{(S, c) \in S_{\Delta}^{(m)}(\Gamma) \times \mathbb{C}^{p}}\|\eta-(S, c)\|_{Y_{m}} \\
& \quad \\
& \leq C(s) \delta_{\Delta}^{s-m}\left\|\left(\partial_{t} h, g\right)\right\|_{\mathcal{H}_{+}^{s}\left(\Gamma_{D}, \Gamma_{N}\right)} \delta_{\Delta}^{m-k}\|\eta\|_{Y_{2 m-k}} \\
& \leq C C(s) \delta_{\Delta}^{s-k}\left\|\left(u_{0}, u_{1}\right)\right\|_{H^{s+1}\left(\Gamma_{D}\right) \times H^{s}\left(\Gamma_{N}\right)}\|\xi\|_{\mathcal{H}_{+}^{k}\left(\Gamma_{D}, \Gamma_{N}\right)^{\prime}}
\end{aligned}
$$

for every $\xi \in \mathcal{H}_{+}^{k}\left(\Gamma_{D}, \Gamma_{N}\right)^{\prime}$. Taking the supremum of the left hand side with respect to the normed elements of $\mathcal{H}_{+}^{k}\left(\Gamma_{D}, \Gamma_{N}\right)^{\prime}$, we obtain the required estimate.

\section{Numerical Results}

We have performed some numerical computations to test the efficiency of the methods. We consider an interior mixed Dirichlet-Neumann problem in the Lshaped region defined in the Figure 1. The sizes of the sides are 1 and 2 . The boundary conditions are also specified in the same figure. This example has been considered in [3, where Galerkin methods are considered.

Solving the same mixed problem with 0 as Neumann data, 1 as Dirichlet data and using Proposition 6, we can compute the Green function and the mixed capacity. Figure 2 represent the functions $\left(\partial_{t} G_{\mid \Gamma_{D}}, \partial_{\nu} G_{\mid \Gamma_{N}}\right)$ on the boundary. We have taken 100 points on each side, and the origin corresponds to the obtuse angle of the polygon. This is a typical behavior for the boundary unknowns of the problem. Of course, the singular terms at the corners are explicitly known. In this case, the most singular one is $x^{-2 / 3}$, where $x$ is the distance to a corner. The value of the mixed capacity is $\gamma=1.19761 \ldots$

The numerical computation of the integrals, which is the most time consuming task, can be performed using high order Gaussian type methods. Indeed, all 


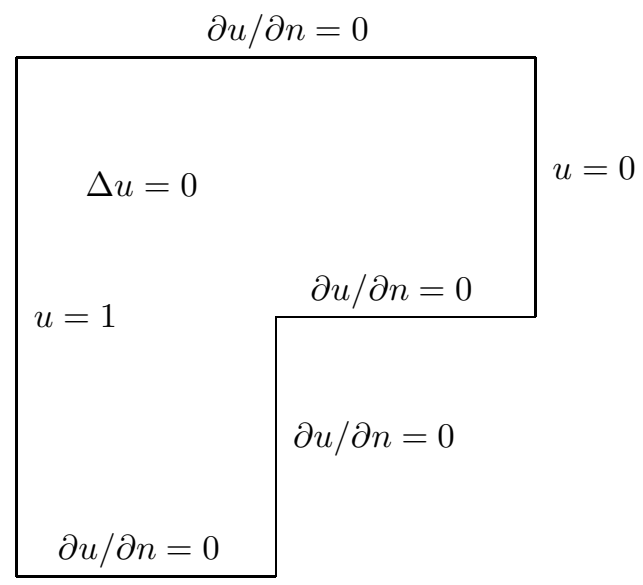

FiguRE 1. L-shaped domain

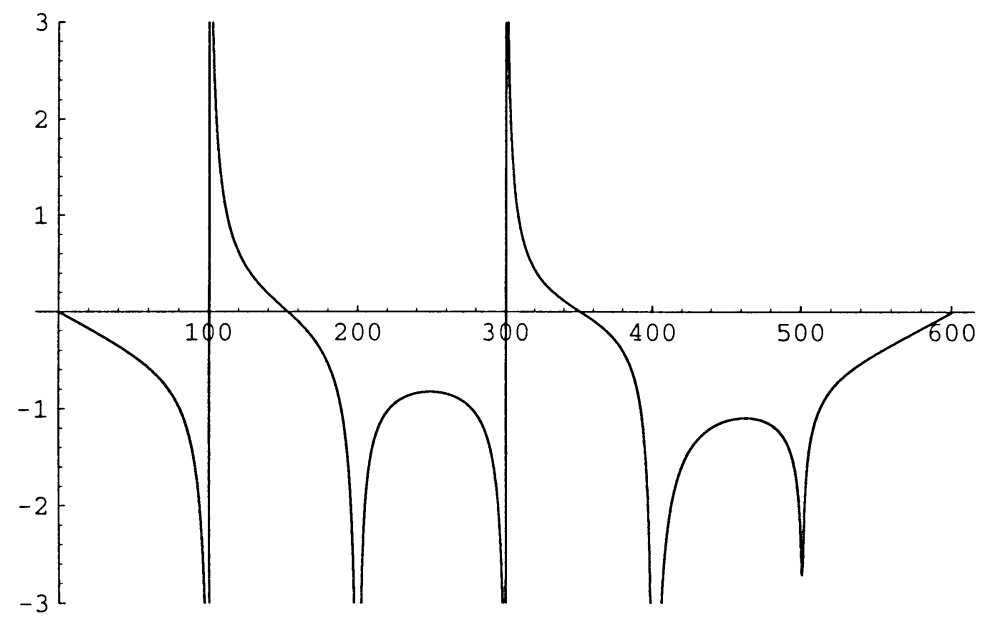

FiguRE 2. Boundary unknown for the Green function

the derivatives involved in the methods can be reduced explicitly to simple integrals, and the functions that we have to integrate are smooth or present a known singularity.

For example, we have the following formulas. Let $\alpha \in e_{\omega, e}^{(p)} \cup e_{\omega, o}^{(p)}$ and $g(x)=$ $x^{\alpha} \chi(x)$. With the - sign if $\alpha \in e_{\omega, e}^{(p)}$ and the + sign in the other case, we have

$$
\frac{1}{\ell !} D^{\ell}(I \mp N) g(0)= \begin{cases} \pm \frac{\sin ((\ell+1) \omega)}{\pi(\alpha-\ell)} \int_{0}^{+\infty} x^{\alpha-\ell} D \chi(x) d x & \text { if } \quad \alpha \neq \ell \\ 1 \mp \frac{\pi-\omega}{\pi} \cos ((\ell+1) \omega) & \text { if } \quad \alpha=\ell\end{cases}
$$


Let $\alpha \in e_{\omega, e}^{(m)} \cup e_{\omega, o}^{(m)}$ and $g(x)=x^{\alpha} \chi(x)$. With the $+\operatorname{sign}$ if $\alpha \in e_{\omega, e}^{(m)}$ and the sign in the other case, we have

$$
\frac{1}{\ell !} D^{\ell}(I \pm W) g(0)= \begin{cases} \pm \frac{\cos ((\ell+1) \omega)}{\pi(\alpha-\ell)} \int_{0}^{+\infty} x^{\alpha-\ell} D \chi(x) d x & \text { if } \quad \alpha \neq \ell \\ 1 \pm \frac{\pi-\omega}{\pi} \sin ((\ell+1) \omega) & \text { if } \quad \alpha=\ell\end{cases}
$$

Let us prove (16). The Mellin transform of a function $f \in \bar{C}_{0}^{\infty}\left(\mathbb{R}_{+}\right)$extends as a meromorphic function in $\mathbb{C}$ with simple poles at $0,-1,-2, \ldots$ This follows from the identity

$$
z(M f)(z)=-M(D f)(z+1)
$$

The residue at $-\ell$ is $D^{\ell} f(0) / \ell$ !. By density, this remains true for any $f \in H^{s}\left(\mathbb{R}_{+}\right)$ if $s>1 / 2+\ell$; see Theorem 4 in [14] or Theorem 1.1.23 in [17. By Proposition 8 $(I \mp N) g$ belongs to $H^{s}\left(\mathbb{R}_{+}\right)$for any $s \geq 0$. Using (11) and (12), we get

$$
\begin{aligned}
\frac{1}{\ell !} D^{\ell}(I & \mp N) g(0) \\
& =\operatorname{Res}_{z=-\ell}(M(I \mp N) g)(z) \\
& =\operatorname{Res}_{z=-\ell}\left[\left(1 \mp \frac{\sin ((z-1)(\pi-\omega))}{\sin (\pi(z-1))}\right) M g(z)\right] \\
& =\operatorname{Res}_{z=-\ell}\left[\left(1 \mp \frac{\sin ((z-1)(\pi-\omega))}{\sin (\pi(z-1))}\right) \frac{1}{z+\alpha} \int_{0}^{+\infty} x^{z+\alpha}(-D \chi)(x) d x\right] \\
& = \pm \frac{\sin ((\ell+1) \omega)}{\pi(\alpha-\ell)} \int_{0}^{+\infty} x^{\alpha-\ell} D \chi(x) d x
\end{aligned}
$$

if $\alpha \neq \ell$. If $\alpha=\ell$, the denominator has a double root at $-\ell$, but the numerator also vanishes and we still have a simple pole which gives the other expression since $\chi(0)=1$. We proceed in the same way for (17).

Tables 1 and 2 present results on the experimental rate of convergence obtained. The boundary unknowns involve singular functions which belong to $H^{-1 / 2}$ but not to $L^{2}$. To obtain finite norms of $L^{2}$ and $H^{1}$ type, we use weighted spaces. The weights chosen are of the form $(s(L-s))^{\alpha}$, where $s$ is the $\operatorname{arc}$ length on a side and $L$ the corresponding length. Of course, the norms depend on $\alpha$, but the computations show that the rates of convergence are essentially independent of $\alpha$.

We present estimates for $\alpha=1 / 2$ in the case of the $L^{2}$-norm. In the case of the $H^{1}$-norm, the derivative leads to even stronger singularities, and we use $\alpha=3 / 2$. These exponents make the integrals finite, but are strictly smaller than the exponent which defines the most singular functions occurring in the solution.

The theoretical rates of convergence appear somewhat pessimistic except in the case of the $L^{2}$-type norm for the linear splines. Even in this case the $H^{1}$-type norm has a very good behavior. A possible explanation for this improvement is that the theoretical rates of convergence are limited only by the approximation of the first missed singular function in the corners. This is a small region, and outside the corners we have a slightly larger order corresponding to approximation of smooth functions by splines.

Computation of the interior potential is of course one essential goal of the method. We have tested the convergence of (3) to the solution at the interior 
TABLE 1. L-shaped polygon, linear splines

\begin{tabular}{|c||c|c||c|c|}
\hline$n$ & $L^{2}$-norm & eoc & $H^{1}$-norm & eoc \\
\hline 4 & $8.27 \mathrm{e}-2$ & & $3.37 \mathrm{e}-1$ & \\
8 & $2.04 \mathrm{e}-2$ & 2.02 & $1.63 \mathrm{e}-1$ & 1.05 \\
16 & $5.29 \mathrm{e}-3$ & 1.94 & $8.29 \mathrm{e}-2$ & 0.97 \\
32 & $1.77 \mathrm{e}-3$ & 1.58 & $4.16 \mathrm{e}-2$ & 0.99 \\
48 & $1.01 \mathrm{e}-3$ & 1.38 & $2.78 \mathrm{e}-2$ & 1.00 \\
64 & $6.99 \mathrm{e}-4$ & 1.29 & $2.08 \mathrm{e}-2$ & 1.00 \\
96 & $4.39 \mathrm{e}-4$ & 1.15 & $1.39 \mathrm{e}-2$ & 1.00 \\
128 & $3.08 \mathrm{e}-4$ & 1.23 & $1.04 \mathrm{e}-2$ & 1.00 \\
\hline
\end{tabular}

TABLE 2. L-shaped polygon, cubic splines

\begin{tabular}{|c||c|c||c|c|}
\hline$n$ & $L^{2}$-norm & eoc & $H^{1}$-norm & eoc \\
\hline 4 & $4.06 \mathrm{e}-2$ & & $1.38 \mathrm{e}-1$ & \\
8 & $1.37 \mathrm{e}-3$ & 4.89 & $1.35 \mathrm{e}-2$ & 3.35 \\
16 & $4.26 \mathrm{e}-5$ & 5.01 & $8.14 \mathrm{e}-4$ & 4.05 \\
32 & $2.25 \mathrm{e}-6$ & 4.24 & $8.79 \mathrm{e}-5$ & 3.21 \\
48 & $4.12 \mathrm{e}-7$ & 4.19 & $2.45 \mathrm{e}-5$ & 3.15 \\
64 & $1.31 \mathrm{e}-7$ & 3.98 & $1.02 \mathrm{e}-5$ & 3.07 \\
96 & $2.63 \mathrm{e}-8$ & 3.96 & $2.98 \mathrm{e}-6$ & 3.03 \\
128 & $8.05 \mathrm{e}-9$ & 4.12 & $1.25 \mathrm{e}-6$ & 3.02 \\
\hline
\end{tabular}

TABLE 3. Interior potential

\begin{tabular}{|c||c|c||c|c|}
\hline$n$ & linear spline & eoc & cubic spline & eoc \\
\hline 4 & $2.92 \mathrm{e}-3$ & & $1.66 \mathrm{e}-4$ & \\
8 & $5.83 \mathrm{e}-4$ & 2.33 & $3.64 \mathrm{e}-6$ & 5.50 \\
12 & $3.20 \mathrm{e}-4$ & 1.48 & $1.90 \mathrm{e}-7$ & 7.29 \\
16 & $2.31 \mathrm{e}-4$ & 1.13 & $5.62 \mathrm{e}-8$ & 4.23 \\
24 & $1.37 \mathrm{e}-4$ & 1.28 & $9.66 \mathrm{e}-9$ & 4.34 \\
32 & $9.72 \mathrm{e}-5$ & 1.20 & $3.15 \mathrm{e}-9$ & 3.89 \\
48 & $6.07 \mathrm{e}-5$ & 1.16 & $4.79 \mathrm{e}-10$ & 4.64 \\
\hline
\end{tabular}

point $(-0.2,0.6)$ when $(g, h)$ is replaced by the numerical approximations. The results are presented in Table 3 and are also in good agreement with the theoretical estimations.

Some parts of this work were performed when the author was visiting the WIAS in Berlin. The author would like to thank Prof. S. Prössdorf and J. Elschner for this opportunity. He also thanks the referee for valuable remarks and comments.

\section{REFERENCES}

1. C.A. Berenstein and R. Gay, Complex variables, an introduction, Graduate Texts in Mathematics, 125, 1991, Springer. MR 92f:30001

2. M. Costabel, Boundary integral operators on Lipschitz domains: elementary results, SIAM J. Math. Anal. 19 (1988), 613-626. MR 89h:35090

3. M. Costabel and E. Stephan, Boundary integral equations for mixed boundary value problems in polygonal domains and Galerkin approximation, Banach Center Publications 15 (1985), 175-251. MR 88f:35037

4. M. Costabel and E. Stephan, On the convergence of collocation methods for boundary integral equations on polygons, Math. Comp. 49 (1987), 461-478. MR 88j:65292

5. M. Costabel and E. Stephan, Duality estimates for the numerical solution of integral equations, Numer. Math. 54 (1988), 339-353. MR 90b:65245 
6. R. Dautray et J.-L. Lions, Analyse mathématique et calcul numérique pour les sciences et les techniques, 4, Méthodes variationnelles, Masson, 1988. MR 89m:00002

7. J. Elschner and I. Graham, An optimal order collocation method for first kind boundary integral equations on polygons, Numer. Math. 70 (1995), 1-31. MR 95m:65215

8. Elschner J., Jeon Y., Sloan I. H. and Stephan E.P.: The collocation method for mixed boundary value problem on domains with curved polygonal boundaries. Numer. Math. 76 (1997), 355381. MR 98h:65055

9. Gaier D., Integralgleichungen erster Art und konforme Abbildung, Math. Z. 147 (1976), 113129. MR 53:786

10. Grisvard P., Elliptic problems in nonsmooth domains. Monographs and Studies in Mathematics 24, Pitman, 1985. MR 86m:35044

11. Hardy G.H., Littlewood J.E. and Pólya G., Inequalities. Cambridge University Press, 1952. MR 13:727e

12. D.S. Jerison and C.E. Kenig, The Dirichlet problem in nonsmooth domains, Ann. of Math. 113 (1981), 367-382. MR 84j:35076

13. D.S. Jerison and C.E. Kenig, The Neumann problem on Lipschitz domains, Bull. Amer. Math. Soc. 4 (1981), 203-207. MR 84a:35064

14. P. Laubin, High order convergence for collocation of second kind boundary integral equations on polygons, Numer. Math. 79 (1998), 107-140. MR 99b:65156

15. P. Laubin and M. Baiwir, Spline collocation for a boundary integral equation on polygons with cuts, Siam. J. Numer. Anal., 35 (1998), 1452-1474. MR 99a:65165

16. S. Prössdorf and B. Silbermann, Numerical analysis for integral and related operator equations, Birkhäuser, Operator Theory: Advances and Applications 52, 1991. MR 94f:65126

17. B.-W. Schulze, Pseudo-differential boundary value problems, conical singularities and asymptotics, Akademie Verlag, Mathematical Topics, Vol. 4, 1994. MR 95e:58172

18. G. Verchota, Layer potentials and regularity for the Dirichlet problem for Laplace's equation in Lipschitz domains, J. Funct. Anal. 59 (1984), 572-611. MR 86e:35038

19. W.L. Wendland, E. Stephan and G.C. Hsiao, On the boundary equation method for the plane mixed boundary value problem of the laplacian, Math. Meth. in the Appl. Sci. I, 1979, 265-321. MR 82e:31003

20. W.L. Wendland, Boundary element methods for elliptic problems. in Mathematical theory of finite and boundary elements methods, DMV Seminar, Band 15, Birkhäuser, 1990. MR 92f:65004

Université de Liège, Institut de Mathématique, Grande Traverse 12, B-4000 Liège, BELGIUM

E-mail address: P.Laubin@ulg.ac.be 